\title{
Strategic production modeling for defective items with imperfect inspection process, rework, and sales return under two-level trade credit
}

\author{
Aditi Khanna, Aakanksha Kishore and Chandra K. Jaggi*
}

Department of Operational Research, Faculty of Mathematical Sciences, New Academic Block, University of Delhi, Delhi-110 007, India

CH R O N I C L E

\section{Article history:}

Received April 42016

Received in Revised Format

June 162016

Accepted July 82016

Available online

July 102016

Keywords:

Inventory

Production

Imperfect items

Inspection

Reworking

Two-stage trade credit \begin{abstract}
A B S T R A C T
Quality decisions are one of the major decisions in inventory management. It affects customer's demand, loyalty and customer satisfaction and also inventory costs. Every manufacturing process is inherent to have some chance causes of variation which may lead to some defectives in the lot. So, in order to cater the customers with faultless products, an inspection process is inevitable, which may also be prone to errors. Thus for an operations manager, maintaining the quality of the lot and the screening process becomes a challenging task, when his objective is to determine the optimal order quantity for the inventory system. Besides these operational tasks, the goal is also to increase the customer base which eventually leads to higher profits. So, as a promotional tool, trade credit is being offered by both the retailer and supplier to their respective customers to encourage more frequent and higher volume purchases. Thus taking into account of these facts, a strategic production model is formulated here to study the combined effects of imperfect quality items, faulty inspection process, rework process, sales return under two level trade credit. The present study is a general framework for many articles and classical EPQ model. An analytical method is employed which jointly optimizes the retailer's credit period and order quantity, so as to maximize the expected total profit per unit time. To study the behavior and application of the model, a numerical example has been cited and a comprehensive sensitivity analysis has been performed. The model can be widely applicable in manufacturing industries like textile, footwear, plastics, electronics, furniture etc.
\end{abstract}

\section{Introduction}

Quality revolves around the concept of meeting or exceeding customer expectation applied to the product and service. Achieving high quality is an ever changing, or continuous process, therefore management is constantly working to improvise quality, so as to serve their customers with good quality products. So, it becomes inevitable to reduce or remove the defects by screening the complete lot before sale. In view of this, researchers have lately shown efforts to develop EOQ and EPQ model for the imperfect quality items. However, the beginning of research on EPQ can be dated back a century ago and was projected by Taft (1918). Porteus (1986), Rosenblatt and Lee (1986), Lee (1987), Schwaller (1988), Zhang and 
Gherchak (1990) were the first few researchers to study the effect of imperfect quality items on EOQ and EPQ models. Furthermore, Salameh and Jaber (2000) carried the research by considering that the whole lot contains a random percentage of defective items with known p.d.f. They also assumed that whole lot goes through $100 \%$ screening process and the sorted out defective items are sold as a single batch at a discounted price. Later, Sana (2010) examined the production in imperfect quality scenario in which the production shifts from "in control" to "out of control" state.

It is again impractical to assume that the inspection process is also perfect. Due to certain human errors, the inspection process leads to errors namely Type-I and Type-II. Due to Type-I error, non-defective items are classified as defective and due to Type-II error, defective items are classified as non-defective. This not only leads to customer dissatisfaction but also sales return bringing inconvenience and frustration to the customers. For the compensation of monetary losses, all the defective items instead of simply discarding can be reworked after inspection process and again treated as perfect items. This has invited many researchers to study the EPQ model extensively under real life situations. Raouf et al. (1983) were among the initial researchers to have inspection errors as a feature in their study. Duffua and Khan (2005) suggested inspection plans for the mistakes committed by the inspector. Papachristos and Konstantaras (2006) emphasized on the issue of non-shortages in inventory models with imperfect quality. Referring to the models of Salameh and Jaber (2000), they pointed out that the conditions proposed as sufficient ones to guarantee that shortages will not occur and cannot really ensure it. Yoo et al. (2009) extended the research by adding defect sales and two disposition methods in their formulation. Khan and Jaber (2011) took similar approach as that of Salameh and Jaber (2000), to reach optimal solution in imperfect quality environment. One of the earliest researchers in production models who considered rework processes was Schrady (1967). Hayek and Salameh (2001) threw light on effect of defective items produced on finite production model. Chiu (2003) developed EPQ model with the assumption that not all of the defectives are repairable and a proportion goes to scrap and will not be reworked. A similar model considering service level constraints with rework was developed by Chiu et al. (2007). Lately, Liu et al. (2009) analyzed the number of production and rework setups used in one cycle; as well as their sequence and optimal production quantity in each setup. Cardenas-Barron (2009) also developed an EPQ model with rework by using a planned backorder. Recently, Chung (2011) revisited the work of Cardenas-Barron to develop a necessary and sufficient condition for the optimal solution. Yoo et al. (2012) developed imperfect-quality inventory models for various inspection options i.e. sampling inspection, entire lot screening and no inspection, under one-time improvement investment in production and inspection reliability. Recently, Wee and Widyadana (2013) studied human errors in inspection and showed the significance of rework and preventive maintenance on optimal time. Further Sarkar et al. (2014) revisited the EPQ model with rework process at a single stage manufacturing system with planned backorders, providing a closed form solution of three different inventory models with three different distribution density functions. Jaggi et al. (2015) explored the effect of deterioration on two warehouse inventory model in imperfect quality scenario. Very recently, Jaggi et al. (2016) have performed elaborated work on imperfect production, inspection and rework process altogether. The authors have developed a mathematical model with the incorporation of five random variables along with the condition of shortages.

Furthermore, in order to survive in this set-up of imperfect productions, many businesses lend loan without interest to their customers as a promotional strategy to increase profitability. Now owing to this trade credit policy, the suppliers do not require to be paid immediately and may agree a delay in payment for goods and services already delivered. Until the expiration of the credit period, the creditors can generate revenue by selling off the items bought on credit and investing the sum in an interest bearing account. Interest is charged if the account is not settled by the end of credit period. In view of this, Haley and Higgins (1973) were the first to consider economic order quantity under permissible delay in payment. Goyal (1985) considered a similar problem including different interest rates before and after the expiration of credit periods. Aggarwal and Jaggi (1995) extended Goyal's model by considering exponential deterioration rate under trade credit. Kim et al. (1995) examined the effect of credit period 
to increase wholesaler's profits with demand as a function of price. Jamal et al. (1997) also generalized Goyal's model to allow for shortages. Teng (2002) further analyzed Goyal's model to include that it is more profitable to order less quantity and make use for permissible delay more frequently.

In today's competition-driven world, with the purpose of increasing profit, the retailer also gives some permissible delay in payment to his own customers. When both supplier and retailer offer credit period to their respective customers, it is termed as two stage trade credit. This not only indicates the seller's faith in the buyer, but also reflects buyer's power to purchase now without immediate payment. Researchers have lately shown efforts in developing two stage trade credit policies. Jaggi et al. (2008) formulated an EOQ model under two-level trade credit policy with credit linked demand. Ho et al. (2008), Teng and Chang (2009) also gave replenishment decisions under two stage trade credits. Thangam and Uthayakumar (2009) extended Jaggi et al. (2008) for perishable items when demand depends on both selling price and credit period under two-level trade credit policy. Recently, Kreng and Tan (2011) developed a production model for a lot size inventory system with finite production rate and defective items which involve imperfect quality and scrap items under the condition of two-level trade credit policy. Recently, Chung and Liao (2011) gave the simplified solution algorithm for an integrated supplier-buyer inventory model with two-part trade credit in a supply chain system. Ouyang and Chang (2013) together explored the effects of the reworking of imperfect quality items and trade credit on the EPQ model with imperfect product processes and complete backlogging. In this direction, Voros (2013) worked on the production modeling without the constraint of defective items in the model. The paper deals with a version of the economic order and production quantity models when the fraction of defective items is probability variable that either may vary from cycle to cycle, or remains the same as it was in the first period. Another contribution in this field was given by Hsu and Hsu (2013a) who developed an economic order quantity model with imperfect quality items, inspection errors, shortage backordering, and sales returns. A closed form solution is obtained for the optimal order size, the maximum shortage level, and the optimal order/reorder point. He further investigated the scenario in Hsu and Hsu (2013b) model where they study two EPQ models with imperfect production processes and inspection errors. The model focuses on the time factor of when to sell the defective items has a significant impact on the optimal production lot size and the backorder quantity. The results show that if customers are willing to wait for the next production when a shortage occurs, it is profitable for the company to have planned backorders although it incurs a penalty cost for the delay. Very lately, Zhou et al. (2015) considered the combined effect of trade credit, shortage, imperfect quality and inspection errors to establish a synergic economic order quantity model, however, they considered one level trade credit with constant demand and without considering reworking of salvage items. In recent times, Tiwari et al. (2016) discussed the impact of trade credit and inflation on retailer's ordering policies for non-instantaneous deteriorating items in a two-warehouse environment. Same year, Chang et al. (2016) developed a model to study the impact of inspection errors and trade credits on the economic order quantity model for items with imperfect quality.

The formal structure of the present model involves imperfect production process, inspection errors, two disposition methods, two way trade credits and a production model. A strategic production model has been developed where the supplier supplies the raw material in semi-finished state to the manufacturer to procure the items and sell them as finished products to his customers i.e. the retailers. Trade credit policies are used by both i.e. the supplier and the manufacturer for their respective customers as it acts as a promotional tool for their businesses. Another valid assumption considered here is that of no shortages. The proposed model jointly optimizes the retailer's credit period and the lot size by maximizing expected total profit per unit time. A numerical example is provided to demonstrate the applicability of the model and a comprehensive sensitivity analysis also has been conducted to observe the effects of key model parameters on the optimal replenishment policy. The literature has also been presented in tabular form for better comparison of past papers with the present model. 
Table 1

Literature Review

\begin{tabular}{|c|c|c|c|c|c|c|c|}
\hline Papers & $\begin{array}{l}\text { Imperfect } \\
\text { Items }\end{array}$ & $\begin{array}{c}\text { Screening } \\
\text { Process }\end{array}$ & $\begin{array}{c}\text { Screening } \\
\text { Errors }\end{array}$ & Rework & Sales Return & $\begin{array}{c}\text { Trade Credit } \\
\text { Policy }\end{array}$ & Shortages \\
\hline Scrady (1967) & Yes & No & No & Yes & No & No & No \\
\hline Raouf et al. (1983) & Yes & Yes & Yes & No & No & No & No \\
\hline Salameh and Jaber (2000) & Yes & Yes & No & No & No & No & No \\
\hline Duffua and Khan (2005) & Yes & Yes & Yes & No & No & No & No \\
\hline Kim et al. (2009) & Yes & No & No & Yes & No & No & No \\
\hline Khan and Jaber (2011) & Yes & Yes & Yes & No & Yes & No & No \\
\hline Hayek and Salameh (2001) & Yes & Yes & No & Yes & No & No & Yes \\
\hline Chiu (2003) & Yes & Yes & No & Yes & No & No & Yes \\
\hline Chiu (2007) & Yes & Yes & No & Yes & No & No & Yes \\
\hline Liu et al. (2009) & Yes & Yes & No & Yes & No & No & No \\
\hline Chiu (2010) & Yes & Yes & No & Yes & No & No & Yes \\
\hline Kim et al. (2012) & Yes & Yes & No & No & No & No & No \\
\hline Wee (2013) & Yes & Yes & No & Yes & No & No & Yes \\
\hline Sarkar et al. (2014) & Yes & Yes & No & Yes & No & No & Yes \\
\hline Jaggi et al. (2008) & Yes & Yes & No & No & No & Yes & Yes \\
\hline Voros (2013) & Yes & No & No & No & No & No & Yes \\
\hline Hsu (2013a) & Yes & Yes & Yes & No & Yes & No & Yes \\
\hline Zhou et al. (2015) & Yes & Yes & Yes & No & Yes & Yes & No \\
\hline Yoo et al. (2009) & Yes & Yes & Yes & Yes & Yes & No & No \\
\hline Jaggi et al. (2016) & Yes & Yes & Yes & Yes & Yes & No & No \\
\hline This paper & Yes & Yes & Yes & Yes & Yes & Yes & No \\
\hline
\end{tabular}

\section{Assumptions and Notations}

The mathematical model proposed in this paper is based on following assumptions and notations.

1. Demand is a function of retailer's credit period $(N)$. it can be derived as a differential difference equation:

$$
\begin{aligned}
& D(N+1)-D(N)=R[U-D(N)] \\
& \text { Where } \\
& \mathrm{D}=\mathrm{D}(N)=\text { demand as a function of } N \text { per unit time } \\
& \mathrm{U}=\text { maximum demand } \\
& \mathrm{R}=\text { rate of saturation of demand (which can be estimated using the past data) }
\end{aligned}
$$

Keeping other attributes like price, quantity, etc. at constant level and by using initial condition: At $N=0, D(N)=u$ (initial demand), the above differential equation can be solved as:

$D=u(1-R)^{N}+U\left[1-(1-R)^{N}\right]$

i.e.

$$
D=U-(U-u)(1-R)^{N}
$$

2. Time horizon is infinite and insignificant lead time.

3. Production and Inspection processes are not perfect.

4. Screening rate is assumed to be greater than the demand rate so as to avoid stock out conditions.

5. The supplier provides a credit period $(M)$ to the manufacturer, who in turn gives a credit period $(N)$ to the retailer.

6. All the defect returns are received by the end of production process and then sent for rework.

7. In the model, the defect proportion, proportion of Type-I error, proportion of Type-II error can be estimated from the past data. Here, these are assumed to follow Uniform distribution.

\section{Parameters}
$P$
Production rate in units per unit time
$\lambda$
Production rate of imperfect items $\left(=d^{*} P\right)$ in units per unit time
Rework rate in units per unit time 
Decision variables $N$ y

Functions

$f(d)$

$f\left(q_{1}\right)$

$f\left(q_{2}\right)$

$f(r)$

$D(N)$

T.R.

T.C.

T.P. $i$

$Z_{i}(y, N)$

$\mathrm{E}\left[Z_{i}(y, N)\right]$

Production and inspection time

Rework Run time

Cycle length

Remaining time in the cycle i.e. $\left(T-t_{1}-t_{2}\right)$

Proportion of imperfect items

Proportion of non-defective items

Proportion of Type-I imperfection error

Proportion of Type-II imperfection error

Proportion of items sent for rework

Expected value operator

Expected value of $\alpha$

Manufacturer's credit period offered by the supplier to settle his accounts (time unit)

Set-up cost for each production run

Production cost per item $(\$ /$ item $)$

Inspection cost per item $(\$ /$ item $)$

Cost of committing Type-I error $(\$ /$ item)

Cost of committing Type-II error $(\$ /$ item)

Selling price $(\$ /$ item $)$

Salvage cost $(<s)(\$ /$ item $)$

Holding cost per unit item per unit time

Holding cost for each imperfect quality item being reworked per unit time

The max on-hand inventory in units, when the regular process ends

The max on-hand inventory in units, when the rework process ends

Interest earning rate per dollar per unit time per year by the manufacturer

Interest payable rate per dollar per unit in stock per year by the manufacturer

Retailer's credit period offered by the manufacturer to settle his account (time unit) Production lot size for each cycle (in units)

p.d.f. of defective items

p.d.f. of Type-I error

p.d.f. of Type-II error

p.d.f. of items sent for rework

Demand rate, a function of retailer's credit period in units per unit time

Manufacturer's Total revenue

Manufacturer's Total cost

Manufacturer's Total profit for $\mathrm{i}=1,2,3,4,5$ cases

Manufacturer's total profit per unit time which is a function of two variables; $y$ and $N$ for $\mathrm{i}=1,2,3,4,5$ cases

Manufacturer's Expected Total profit per unit time for $\mathrm{i}=1,2,3,4,5$ cases

Optimal values

$D^{*}(N)$

$T^{*}$

$N^{*}$

$y^{*}$

$Z *(y, N)$

$\mathrm{E}\left[Z^{*}(y, N)\right]$

Optimal Demand rate for optimal $N$ per unit time

Optimal cycle length

Optimal credit period for the retailer

Optimal production quantity per cycle

Manufacturer's optimal total profit per unit time

Manufacturer's optimal expected total profit per unit time 


\section{Model Description and Formulation}

When the items are produced within the firm and not purchased from outside to meet the demands, such a process is known as manufacturing process and the goal of most manufacturing firms is to maximize the profit by producing the optimal quantity so that there is no overstocking or under stocking. In a 3 tier supply chain for the production model, the supplier provides raw material to the manufacturer who processes the semi- finished products to procure the finished item ready for selling to his customers i.e. the retailers here. The mathematical model used to assist such firms in maximizing the profit by determining optimal production lot size is called Economic Production Quality (EPQ) model. The production and inspection rate have to be greater than the demand rate for smooth functioning of the system and to avoid shortage conditions, respectively, so it is also called Finite Production model. In any production process due to certain reasons like deterioration, improper transport, weak process control or any other factor, the production may shift to imperfect production process in which not all the items are of good quality. Due to this whole lot goes through the inspection process which is also prone to errors, i.e. Type-I which causes direct loss to the manufacturer by stopping him to generate revenue on full sales since it identifies a perfect item as defective and Type-II inspection error which not only causes monetary losses but also customer dissatisfaction which is more difficult to recover. Due to this error, a defective item is identified as non-defective, thereby passing on to customers and resulting in defect sales return. Production and inspection occur simultaneously. After the end of production process, rework of defective item begins. Not all defective items are sent for rework; some are discarded and sold as scrap. Demand is continuously satisfied from perfect or reworked items. In this paper, a three tier supply chain has been considered, where production and inspection process is not perfect.

In producing lot $y$, due to imperfect production quality, $d$ proportion of defective items are produced with known probability density function $f(d)$. So, lot $y$ has defective items as $d y$ and non-defective items as (1-d)y. Due to imperfect inspection process, there is generation of Type-I and Type-II errors, given their respective proportions of $q_{1}=P_{r}$ (items screened as defects $\mid$ non-defective items) and $q_{2}=P_{r}$ (items not screened as defects $\mid$ defective items $)\left(0<q_{1}<q_{2}<1\right)$ following p.d.f. of $f\left(q_{1}\right)$ and $f\left(q_{2}\right)$ respectively. It is assumed, $q_{1}$ and $q_{2}$ are independent of defect proportions $d$. So, all the items involving inspection errors are determined inter-dependently by $q_{1}, q_{2}$, and $y$. In Type-II error, non-defective items are falsely treated as defects thereby losing an opportunity to make more profit by selling them to customers at selling price $(s)$. Due to Type-I error, $(1-d) q 1 y$ units among the non-defective items of $(1-d) y$ are falsely treated as defects, leaving $(1-r)\left(1-q_{1}\right) y$ units of the non-defects as perfect and ready for sale. In Type-II error, the defective items are wrongly sold to the customers by treating them like perfect or non-defective items, resulting in sales return and loss of goodwill. Due to Type-II error, $d q_{2} y$ units among the defective items of $d y$ are falsely treated as non-defects, leaving $d\left(1-q_{2}\right) y$ units among the defects. Since demand $D(N)$ is satisfied by perfect items only, it becomes practically important to examine imperfect production and inspection process which affects a firm's profitability. After the inspection of the entire lot $y$, all the sorted-out non-defective items are summed as $\left[d_{2} y+(1-d)\left(1-q_{1}\right) y\right]$ which include falsely inspected defects (Type-II error) and successfully inspected non-defects respectively and the total sorted out defective items are summed up as $\left[d\left(1-q_{2}\right) y+q_{1}(1-d) y\right]$ which include successfully inspected defects and falsely inspected non-defects (Type-I error) respectively. Those falsely inspected defects $\left(d q_{2} y\right)$ result in sales return when passed on to customers due to quality dissatisfaction. Two disposition methods are employed to settle out defective items and defect returns. One is rework process and other is discarding them as scrap. The defect returns are assumed to re-enter the inventory cycle continuously like demand and get accumulated over the length of period $T$ so that the defect returns and defective items can be sent for rework together at a constant rate $P_{l}$ in the next cycle after time duration $t_{1}$. Not all the defective items go for the rework process, some are discarded beforehand at a lesser price $v(<s)$. A proportion $r$ is sent for rework, given its p.d.f. $f(r)$. Total items collected for rework and salvage are $\left[d\left(1-q_{2}\right) y+d q_{2} y+q_{1}(1-\right.$ d)y], which include successfully inspected defective items, sales return and falsely inspected non-defects respectively. So, the reworked items are $\left[d+(1-d) q_{1}\right] r y$ and salvaged items are $\left[d+(1-d) q_{1}\right](1-r) y$. Reworked items are treated as good as perfect items and sold at the same selling price $(s)$. Disposing off 
of salvage items occurs at the end of each production and inspection cycle as in Salameh and Jaber (2000).

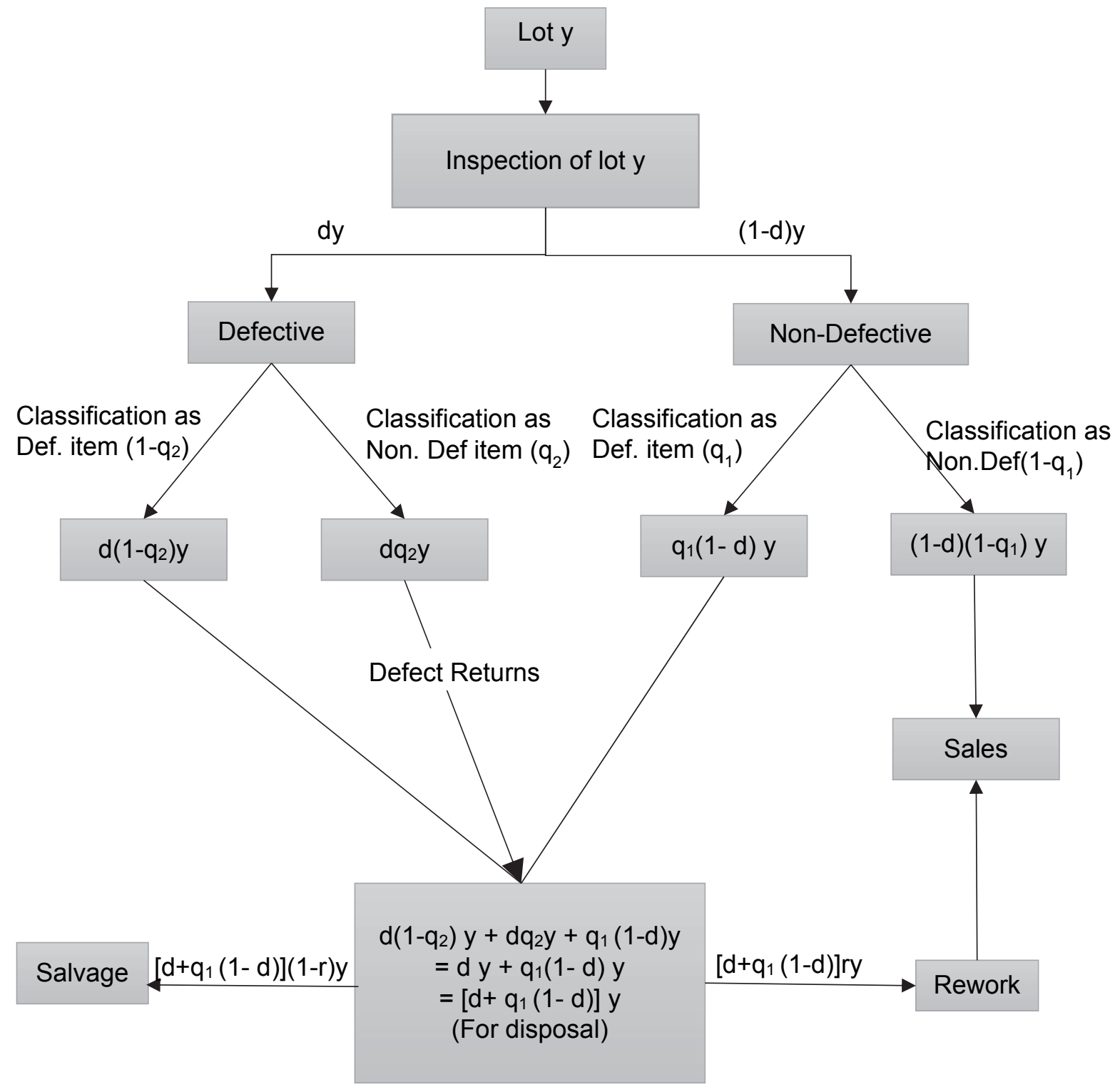

Fig. 1. Flowchart of the processes taking place

It is also assumed that there is a delay in payment allowed up to a credit limit and there is no interest charged within this limit. However if the account is not settled within this limit, there is interest charged beyond the credit point. When both supplier and retailer offer credit period to their respective customers, this is termed as two stage credit policy which has been considered in this paper. Demand is taken as a function of customer's credit period $N$ and supplier's credit period is $M$. Here, $N$ is taken as the second decision variable and has been jointly optimized with $y$. Finally, no shortages are allowed in each cycle $T$. The sequence of all the above described events is shown in Fig. 1. The behavior of the inventory model describing the whole scenario is shown in Fig. 2. The aim of this model is to determine the optimal production lot size $y$ and the retailer's credit period $N$ that maximizes the expected total profit per unit time $(\mathrm{E}[Z(y, N)])$. Various factors contributing to the total profit per unit time (T.P.U.) are: Total Revenue, Total Cost, Interest Earned and Interest Paid. 
(a)

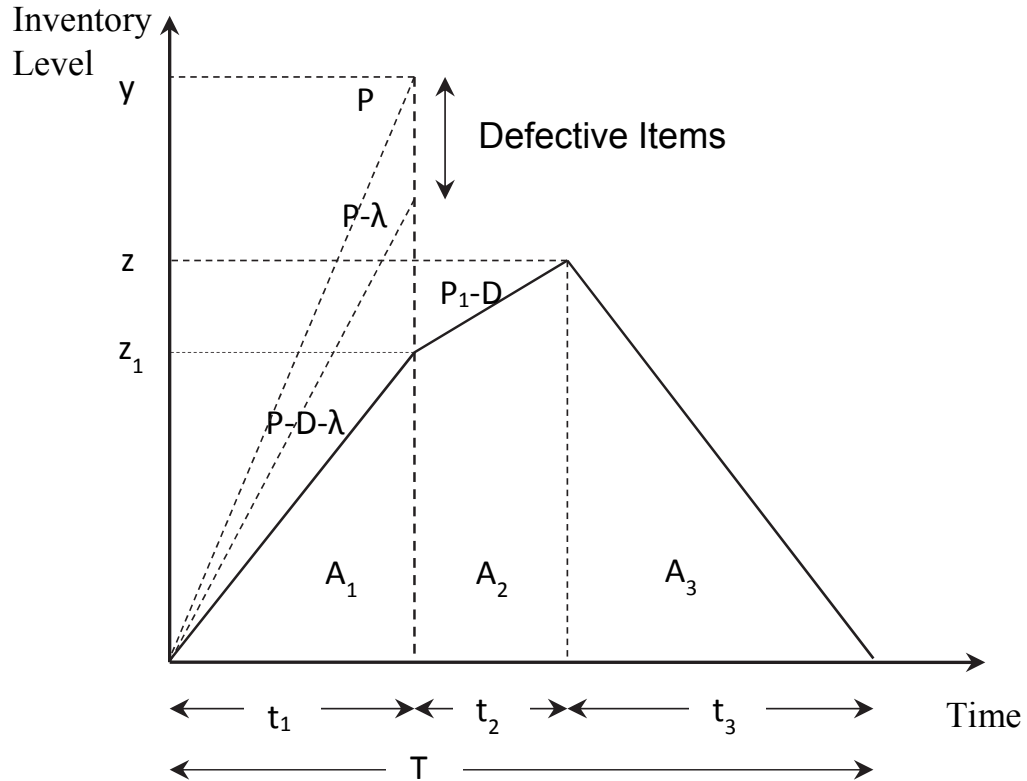

(b) Inventory Level
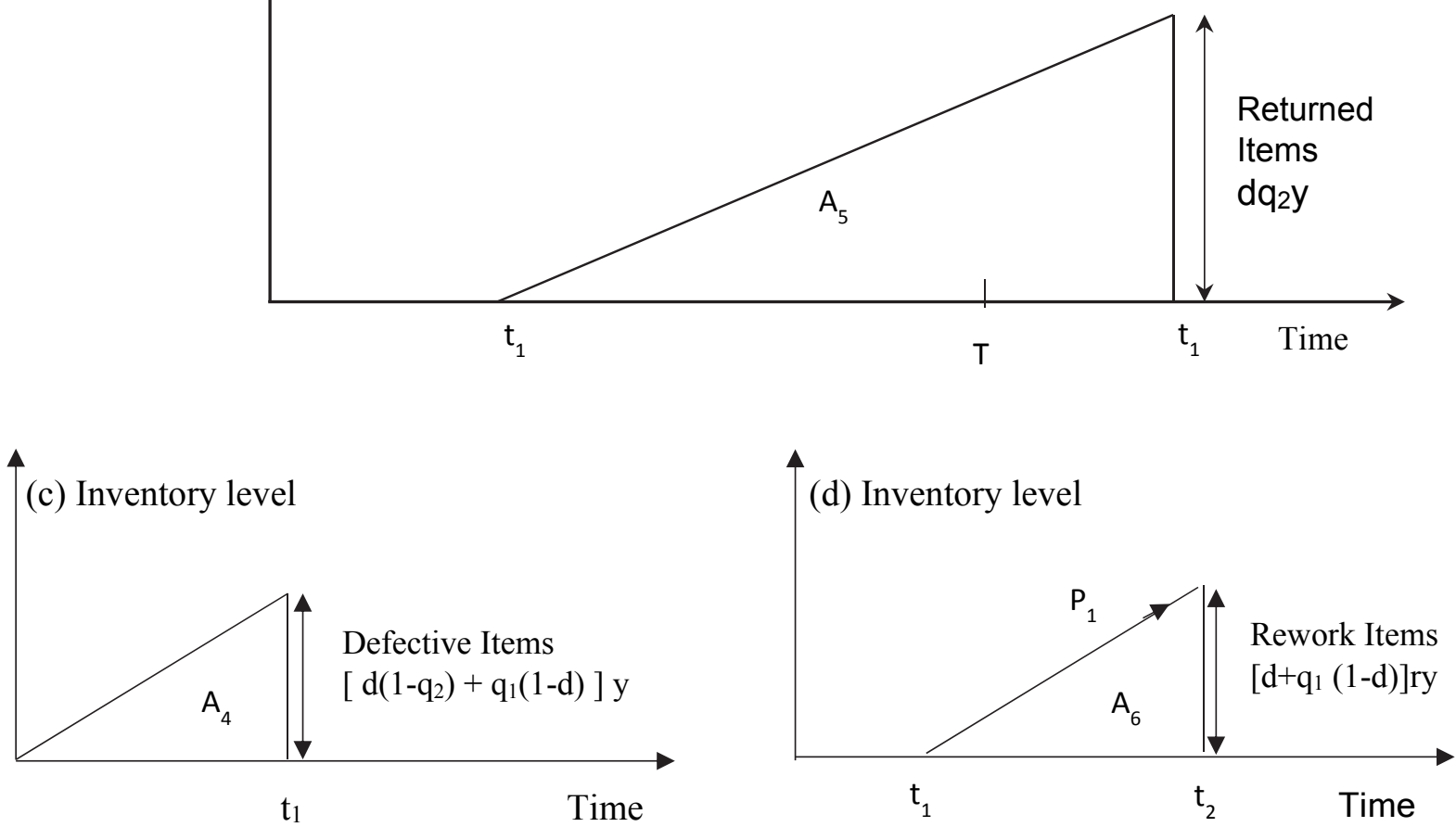

Fig. 2. Inventory behavior of (a) imperfect production and inspection system, (b) defect returns, (c) defective items sorted through inspection process, (d) reworked items

The conditions which conform that there will be no shortage conditions in the model are:

a. Total number of perfect items should be greater than the demand during the inspection period i.e. $\left[d q_{2}+(1-d)\left(1-q_{1}\right)\right] y>D t_{1}$, i.e.

$$
\beta y>D t_{1}
$$


where

$$
\beta=d q_{2}+(1-d)\left(1-q_{1}\right)
$$

$\beta$ being a combination of random variables viz. $d, q_{1}, q_{2}$, is also a random variable.

So,

$$
\mathrm{E}[\beta]=\mathrm{E}\left[d q_{2}\right]+(1-\mathrm{E}[d])\left(1-\mathrm{E}\left[q_{1}\right]\right)
$$

b. Total number of perfect items(including reworked items) after the inspection process should be greater than the demand during rest of the period i.e.

$$
\begin{aligned}
& {\left[d q_{2}+(1-d)\left(1-q_{1}\right)\right] y-D t_{1}+\left[d+q_{1}(1-d)\right] r y \geq D\left(t_{2}+t_{3}\right) \text { i.e. }} \\
& \quad\left(\beta y-D t_{1}+\delta r y\right) \geq D\left(t_{2}+t_{3}\right)
\end{aligned}
$$

where

$$
\delta=\left[d+q_{1}(1-d)\right]
$$

$\delta$ being a combination of random variables viz. $d$, $q_{1}$, is also a random variable.

So,

$$
\mathrm{E}[\delta]=\mathrm{E}[d]+\mathrm{E}\left[q_{1}\right](1-\mathrm{E}[d])
$$

From Fig. 2, some basic formulae are derived:

$$
\begin{aligned}
& \boldsymbol{t}_{\mathbf{1}}=\frac{z_{1}}{P-D-\lambda} \text { Also, } \boldsymbol{t}_{\mathbf{1}}=\frac{y}{P} \\
& \boldsymbol{t}_{\mathbf{2}}=\frac{\left[d+q_{1}(1-d)\right] r y}{P_{1}}=\frac{\delta r y}{P_{1}} \\
& \boldsymbol{t}_{\mathbf{3}}=\frac{z}{D}
\end{aligned}
$$

Using Eq. (6), (7), the values of $z, z_{1}$ are derived:

$$
\begin{aligned}
& \mathbf{z}_{\mathbf{1}}=(P-D-\lambda) t_{1} \\
& \mathbf{z}_{\mathbf{1}}=(P-D-\lambda) \frac{y}{P} \\
& \mathbf{z}=z_{1}+\left(P_{1}-D\right) t_{2} \\
& \mathbf{z}=(P-D-\lambda) \frac{y}{P}+\left(P_{1}-D\right)\left(\frac{\delta r y}{P_{1}}\right)
\end{aligned}
$$

Therefore, from Eq. (6), (7), (8) and (10)

Cycle length $=\Sigma t_{i}, \mathrm{i}=1,2,3$ i.e.

$$
T=\frac{y}{P}+\frac{\left[d+q_{1}(1-d)\right] r y}{P_{1}}+\frac{1}{D}\left[(P-D-\lambda) \frac{y}{P}+\left(P_{1}-D\right)\left(\frac{\text { sry }}{P_{1}}\right)\right]
$$

Also, by following the same procedure as that of Yoo et al. (2009), the cycle length can be obtained from the depletion time of all the serviceable items sold as per demand rate, i.e $\boldsymbol{T}=\frac{\left\{d q_{2}+(1-d)\left(1-q_{1}\right)+\left[d+q_{1}(1-d)\right] r\right\} y}{D}$ 


$$
\boldsymbol{T}=\alpha \frac{y}{D}
$$

where

$$
\alpha=(\beta+\delta r) \text {, say }
$$

$\alpha$ being a combination of random variables viz. $\beta, \delta, r$ is also a random variable.

So,

$$
\mathrm{E}[\alpha]=\mathrm{E}[\beta]+\mathrm{E}[\delta r]
$$

Various components of total revenue are:

$$
\begin{array}{cl}
\text { i. } & \text { Sales revenue of Non-Defective items }=s\left[d q_{2}+(1-d)\left(1-q_{1}\right)\right] y \\
\text { ii. } & \text { Revenue loss from Defect Refund }=s d q_{2} y \\
\text { iii. } & \text { Sales revenue of Reworked Items }=s\left[d+(1-d) q_{1}\right] r y \\
\text { iv. } & \text { Sales revenue of Salvage Items }=v\left[d+(1-d) q_{1}\right](1-r) y
\end{array}
$$

By using Eqs. (14a-14d), we obtain:

Total Revenue (T.R.) = Sales revenue of Non-Defective items - Revenue loss from Defect

Refund + Sales revenue of Reworked Items + Sales revenue of Salvage Items

$$
\begin{aligned}
& =s\left[d q_{2}+(1-d)\left(1-q_{1}\right)\right] y-s d q_{2} y+s\left[d+(1-d) q_{1}\right] r y+v\left[d+(1-d) q_{1}\right](1-r) y \\
& (\boldsymbol{T . R .})=\beta s y-s d q_{2} y+\delta r s y+\delta v(1-r) y
\end{aligned}
$$

Various components of cost function are:

$$
\begin{aligned}
& \text { i. Setup Cost }=K \\
& \text { ii. Purchase Cost }=c y \\
& \text { iii. Inspection Cost }=i y \\
& \text { iv. Cost of committing Type-I error }=C_{r}(1-d) q_{1} y \\
& \text { v. Cost of committing Type-II error }=C_{a} d q_{2} y \\
& \text { vi. Rework Cost }=\left[d+(1-d) q_{1}\right] w r y \\
& \text { vii. Inventory Holding Cost }=h\left\{\frac{z_{1} t_{1}}{2}+\frac{\left(z+z_{1}\right) t_{2}}{2}+\frac{1}{2} z t_{3}+\frac{d q_{2} y T}{2}+\frac{1}{2}[d(1-\right. \\
& \left.\left.\left.q_{2}\right)+q_{1}(1-d)\right] y t_{1}\right\}+h_{1}\left\{\frac{1}{2}\left[d+q_{1}(1-d)\right] r y t_{2}\right\}
\end{aligned}
$$

By using Eqs. (16a-16g), we obtain:

Total Cost (T.C.) = Setup Cost + Purchase Cost + Inspection Cost + Cost of committing Type-I error + Cost of committing Type-II error + Rework Cost + Inventory Holding Cost

$$
\begin{aligned}
& (\text { T.C. })=c y+K+i y+C_{r}(1-d) q_{1} y+C_{a} d q_{2} y+\left[d+(1-d) q_{1}\right] w r y+h\left\{\frac{(P-D-\lambda)\left(\frac{y}{P}\right)^{2}}{2}+\left[\frac{2(P-D-\lambda) y}{P}+\right.\right. \\
& \left.\left(P_{1}-D\right)\left(\frac{\left[d+q_{1}(1-d)\right] r y}{P_{1}}\right)\right]\left(\frac{\left[d+q_{1}(1-d)\right] r y}{P_{1}}\right)+\frac{1}{2 D}\left[\frac{(P-D-\lambda) y}{P}+\left(P_{1}-D\right)\left(\frac{\left[d+q_{1}(1-d)\right] r y}{P_{1}}\right)\right]^{2}+\frac{d q_{2} \alpha y^{2}}{2 D}+\frac{1}{2 P}[d(1- \\
& \left.\left.\left.q_{2}\right)+q_{1}(1-d)\right] y^{2}\right\}+\frac{h_{1}}{2}\left\{\left[d+q_{1}(1-d)\right] r y\right\}^{2}
\end{aligned}
$$


Now, depending upon the value of $M, N$ and $T$, the value of interest earned and interest paid is calculated for five distinct possible cases $Z_{j}(y, N) ; \mathrm{j}=1,2,3,4,5 \mathrm{viz}$.

Case (i) $N \leq M \leq t_{1}+N \leq T+N$

Case (ii) $N \leq t_{1}+N \leq M \leq T+N$

Case (iii) $N \leq t_{1}+t_{2}+N \leq M \leq T+N$

Case (iv) $T+N \leq M$

Case (v) $M \leq N \leq T+N$

Case (i) $\quad N \leq M \leq t_{1}+N \leq T+N$

From the Fig. 3, it is clearly visible that the interest earning period for the manufacturer is from $N$ to $M$, as he starts getting his actual sales from $N$. At $M$, the manufacturer settles his account with the supplier and arranges for the finances to make the payment to the supplier for the left over stock which are the remaining perfect and reworked items used to satisfy demand and the items for disposal, which include the actual defectives, sales return and falsely sorted defectives. Interest is charged on the unsold items for the time $M$ to $T+N$.

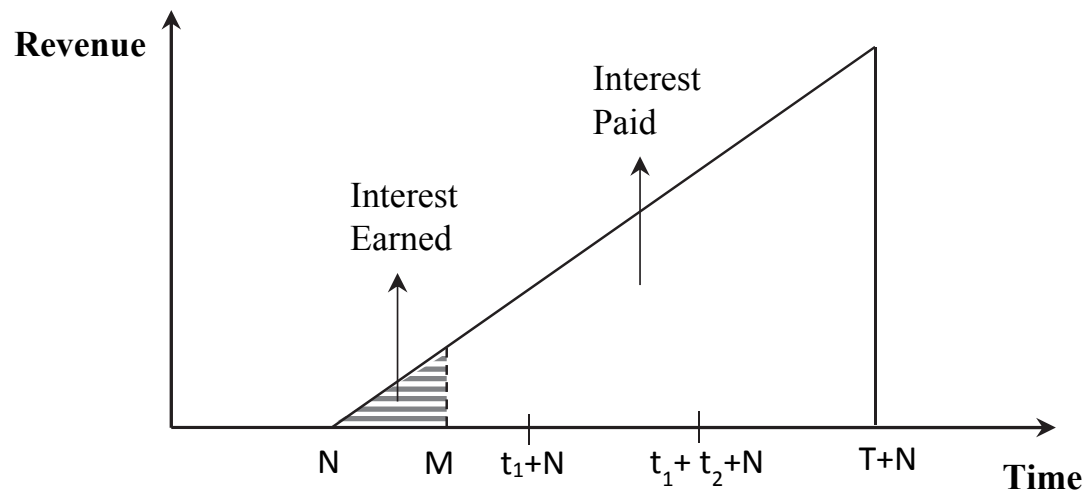

Fig. 3. $N \leq M \leq t_{1}+N \leq T+N$

Interest Earned $\left(I_{e l}\right)=\frac{1}{2} s I_{e} D(M-N)^{2}$

Interest Payable $\left(\boldsymbol{I}_{\boldsymbol{p} I}\right)=c I_{p}\left[\frac{1}{2} D\left(t_{1}+N-M\right)^{2}+\frac{1}{2} D t_{2}{ }^{2}+\frac{1}{2} D t_{3}{ }^{2}\right]+$

$$
\begin{aligned}
& c I_{p}\left[d+q_{1}(1-d)\right](1-r) y\left(t_{1}+N-M\right) \\
& \left(I_{p 1}\right)=c I_{p}\left[\frac{1}{2} D\left(\frac{y}{P}+N-M\right)^{2}+\frac{1}{2} D \frac{\delta^{2} r^{2} y^{2}}{P_{1}{ }^{2}}+\frac{1}{2 D}\left\{\frac{(P-D-\lambda) y}{P}+\left(P_{1}-D\right) \frac{\delta r y}{P_{1}}\right\}^{2}\right]+ \\
& c I_{p} D \delta(1-r) y\left(\frac{y}{P}+N-M\right)
\end{aligned}
$$

Total Profit (T.P.1) $=$ Total Revenue $($ T.R. $)-$ Total Cost (T.C. $)+$ Interest Earned $\left(I_{e l}\right)-$ Interest

Payable $\left(I_{p 1}\right)$

$$
\begin{aligned}
& (\text { T.P.1 })=\beta s y-s d q_{2} y+\delta r s y+\delta v(1-r) y-\left\{c y+K+i y+C_{r}(1-d) q_{1} y+\right. \\
& C_{a} d q_{2} y+\delta w r y+h\left\{\frac{(P-D-\lambda)\left(\frac{y}{P}\right)^{2}}{2}+\left[\frac{2(P-D-\lambda) y}{P}+\left(P_{1}-D\right)\left(\frac{\delta r y}{P_{1}}\right)\right]\left(\frac{\delta r y}{P_{1}}\right)\right\}+ \\
& h\left\{\frac{1}{2 D}\left[\frac{(P-D-\lambda) y}{P}+\left(P_{1}-D\right)\left(\frac{\delta r y}{P_{1}}\right)\right]^{2}+\frac{d q_{2} \alpha y^{2}}{2 D}+\frac{1}{2 P}\left[d\left(1-q_{2}\right)+q_{1}(1-d)\right] y^{2}\right\}+
\end{aligned}
$$




$$
\begin{aligned}
& \left.\frac{h_{1}}{2}(\delta r y)^{2}\right\}+\frac{1}{2} s I_{e} D(M-N)^{2}-c I_{p}\left[\frac{1}{2} D\left(\frac{y}{P}+N-M\right)^{2}+\frac{1}{2} D \frac{\delta^{2} r^{2} y^{2}}{P_{1}{ }^{2}}+\right. \\
& \left.\frac{1}{2 D}\left\{\frac{(P-D-\lambda) y}{P}+\left(P_{1}-D\right) \frac{\delta r y}{P_{1}}\right\}^{2}\right]-c I_{p} D \delta(1-r) y\left(\frac{y}{P}+N-M\right)
\end{aligned}
$$

By using Eq. (12) and Eq. (20), we get:

$$
\begin{aligned}
& \text { Expected Total Profit (E.T.P. 1) }=s y E[\beta]-s y E\left[d q_{2}\right]+s y E[\delta r]+v y E[\delta](1-E[r])- \\
& \left\{c y+K+i y+C_{r} y(1-E[d]) E\left[q_{1}\right]+C_{a} y E\left[d q_{2}\right]+w y E[\delta r]+h\left\{\frac{(P-D-\lambda)}{2}\left(\frac{y}{P}\right)^{2}+\left[2(P-D-\lambda) \frac{y}{P}+\right.\right.\right. \\
& \left.\left.\left(P_{1}-D\right)\left(\frac{y}{P_{1}}\right) E[\delta r]\right]\left(\frac{y}{P_{1}}\right) E[\delta r]\right\}+ \\
& h\left\{\frac{1}{2 D}\left[(P-D-\lambda)\left(\frac{y}{P}\right)+\left(P_{1}-D\right)\left(\frac{y}{P_{1}}\right) E[\delta r]\right]^{2}+E\left[d q_{2} \alpha\right] \frac{y^{2}}{2 D}+\frac{1}{2 P}\left[E[d]\left(1-E\left[q_{2}\right]\right)+E\left[q_{1}\right](1-E[d])\right] y^{2}\right\} \\
& \left.\frac{h_{1} y^{2}}{2} E\left[\delta^{2} r^{2}\right]\right\}+\frac{1}{2} s I_{e} D(M-N)^{2}-c I_{p}\left[\frac{1}{2} D\left(\frac{y}{P}+N-M\right)^{2}+\frac{1}{2} D E\left[\delta^{2} r^{2}\right] \frac{y^{2}}{P_{1}{ }^{2}}+\frac{1}{2 D}\left\{(P-D-\lambda) \frac{y}{P}+\right.\right. \\
& \left.\left.\left(P_{1}-D\right) \frac{y}{P_{1}} E[\delta r]\right\}^{2}\right]-c I_{p} D E[\delta](1-E[r]) y\left(\frac{y}{P}+N-M\right)
\end{aligned}
$$

By using Renewal- reward theorem, we get:

Expected Total Profit per unit time $\left(\mathrm{E}\left[Z_{1}(y, N)\right]\right)=s D+\frac{1}{E[\alpha]}\left[-c D-i D-\frac{K D}{y}-\frac{h(P-D-\lambda) D y}{2 P^{2}}-\right.$

$$
\begin{aligned}
& \left.\frac{h(P-D-\lambda)^{2} y}{2 P^{2}}+\frac{S I_{e} D^{2}(M-N)^{2}}{2 y}-\frac{c I_{p} D^{2}}{2 y}\left(\frac{y}{P}+N-M\right)^{2}-\frac{c I_{p}(P-D-\lambda)^{2} y}{2 P^{2}}\right]-\frac{E[\delta r]}{E[\alpha]}\left[(v+w) D+\frac{2 h(P-D-\lambda) D y}{P P_{1}}+\right. \\
& \left.\frac{2 h(P-D-\lambda)\left(P_{1}-D\right) D y}{P P_{1}}+\frac{c I_{p}(P-D-\lambda)\left(P_{1}-D\right) y}{P P_{1}}-C I_{p} D\left(\frac{y}{P}+N-M\right)\right]-\frac{E\left[\delta^{2} r^{2}\right]}{E[\alpha]}\left[W \frac{h\left(P_{1}-D\right) D y}{P_{1}{ }^{2}}+\frac{h\left(P_{1}-D\right)^{2} y}{2 P_{1}{ }^{2}}+\frac{h_{1} D y}{2 P_{1}}+\right. \\
& \left.\frac{c I_{p} D^{2} y}{2 P_{1}{ }^{2}}+\frac{c I_{p}\left(P_{1}-D\right)^{2} y}{2 P_{1}{ }^{2}}\right]+\frac{E[\delta]}{E[\alpha]}\left[v D-C I_{p} D\left(\frac{y}{P}+N-M\right)\right]-D C_{r} \frac{(1-E[d]) E\left[q_{1}\right]}{E[\alpha]}-D C_{a} \frac{E\left[d q_{2}\right]}{E[\alpha]}-\frac{h y}{2} \frac{E\left[d q_{2} \beta\right]}{E[\alpha]}- \\
& \frac{h y}{2} \frac{E\left[d q_{2} \delta r\right]}{E[\alpha]}-\frac{h D y}{2 P} \frac{E[d]\left(1-E\left[q_{2}\right]\right)+E\left[q_{1}\right](1-E[d])}{E[\alpha]}
\end{aligned}
$$

where, $E[$.$] denotes the expected value.$

Case (ii) $\quad N \leq t_{1}+N \leq M \leq T+N$

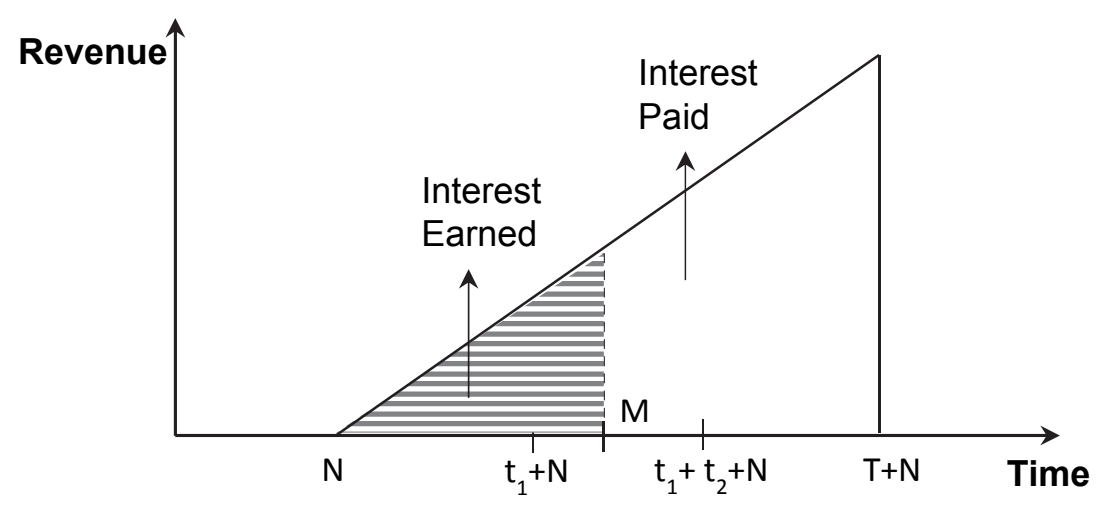

Fig. 4. $N \leq t_{1}+N \leq M \leq T+N$

As visible in Fig. 4, the manufacturer earns interest on sales revenue from $N$ to $M$, as he gets his first delivery of cash at $N$. These items include the defect returns from the market for the time period $\left(t_{1}+\right.$ 
$N, M)$. Since the items for disposal have also been sold at $t_{1}$, he earns additional interest on these items also. The whole amount is accumulated in an interest bearing account till the time $M$. After the settlement of manufacturer's account with the supplier at $M$, the unsold items have to be financed by the manufacturer on his own to make the payment to the supplier which constitutes the remaining perfect items, reworked items used to satisfy the demand.

$$
\begin{gathered}
\text { Interest Earned }\left(\boldsymbol{I}_{e 2}\right)=s I_{e}\left[\frac{1}{2} D t_{1}{ }^{2}+D t_{1}\left(M-t_{1}-N\right)+\frac{1}{2} D\left(M-t_{1}-N\right)^{2}\right]+ \\
v I_{e}\left[d+q_{1}(1-d)\right](1-r) y\left(M-t_{1}-N\right) \\
\left(I_{e 2}\right)=s I_{e}\left[\frac{1}{2} D\left(\frac{y}{P}\right)^{2}+\frac{D y}{P}\left(M-\frac{y}{P}-N\right)+\frac{1}{2} D\left(M-\frac{y}{P}-N\right)^{2}\right]+v I_{e} \delta(1-r) y\left(M-\frac{y}{P}-N\right)
\end{gathered}
$$

Interest Payable $\left(\boldsymbol{I}_{p 2}\right)=c I_{p}\left[\frac{1}{2} D\left(t_{1}+t_{2}+N-M\right)^{2}+\frac{1}{2} D t_{3}^{2}\right]$

$$
\left(\boldsymbol{I}_{p 2}\right)=C I_{p}\left[\frac{1}{2} D\left(\frac{y}{P}+\frac{\delta r y}{P_{1}}+N-M\right)^{2}+\frac{1}{2 D}\left\{\frac{(P-D-\lambda) y}{P}+\frac{\left(P_{1}-D\right) \delta r y}{P_{1}}\right\}^{2}\right]
$$

Total Profit (T.P.2) $=$ Total Revenue (T.R.) - Total Cost (T.C.) + Interest Earned $\left(I_{e 2}\right)-$ Interest

\section{Payable $\left(I_{p 2}\right)$}

Total Profit (T.P. 2) $=\beta s y-s d q_{2} y+\delta r s y+\delta v(1-r) y-\left\{c y+K+i y+C_{r}(1-\right.$

$$
\begin{aligned}
& \text { d) } q_{1} y+C_{a} d q_{2} y+\delta w r y+h\left\{\frac{(P-D-\lambda)\left(\frac{y}{P}\right)^{2}}{2}+\left[\frac{2(P-D-\lambda) y}{P}+\left(P_{1}-D\right)\left(\frac{\delta r y}{P_{1}}\right)\right]\left(\frac{\delta r y}{P_{1}}\right)+\right. \\
& \left.\frac{1}{2 D}\left[\frac{(P-D-\lambda) y}{P}+\left(P_{1}-D\right)\left(\frac{\delta r y}{P_{1}}\right)\right]^{2}+\frac{d q_{2} \alpha y^{2}}{2 D}+\frac{1}{2 P}\left[d\left(1-q_{2}\right)+q_{1}(1-d)\right] y^{2}\right\}+ \\
& \left.\frac{h_{1}}{2}(\delta r y)^{2}\right\}+s I_{e}\left[\frac{1}{2} D\left(\frac{y}{P}\right)^{2}+\frac{D y}{P}\left(M-\frac{y}{P}-N\right)+\frac{1}{2} D\left(M-\frac{y}{P}-N\right)^{2}\right]+v I_{e} \delta(1-r) y(M- \\
& \left.\frac{y}{P}-N\right)-c I_{p}\left[\frac{1}{2} D\left(\frac{y}{P}+\frac{\delta r y}{P_{1}}+N-M\right)^{2}+\frac{1}{2 D}\left\{\frac{(P-D-\lambda) y}{P}+\frac{\left(P_{1}-D\right) \delta r y}{P_{1}}\right\}^{2}\right]
\end{aligned}
$$

By using Eq. (12) and Eq. (25), we get:

$$
\begin{aligned}
& \text { Expected Total Profit (E.T.P. })=s y E[\beta]-s y E\left[d q_{2}\right]+s y E[\delta r]+v y E[\delta](1-E[r])-\left\{c y+K+i y+C_{r} y(1-\right. \\
& \mathrm{E}[d]) E\left[q_{1}\right]+C_{a} y E\left[d q_{2}\right]+w y E[\delta r]+h\left\{\frac{(P-D-\lambda)}{2}\left(\frac{y}{P}\right)^{2}+\left[2(P-D-\lambda) \frac{y}{P}+\left(P_{1}-D\right)\left(\frac{y}{P_{1}}\right) E[\delta r]\right]\left(\frac{y}{P_{1}}\right) E[\delta r]\right\}+ \\
& h\left\{\frac{1}{2 D}\left[(P-D-\lambda)\left(\frac{y}{P}\right)+\left(P_{1}-D\right)\left(\frac{y}{P_{1}}\right) E[\delta r]\right]^{2}+E\left[d q_{2} \alpha\right] \frac{y^{2}}{2 D}+\frac{1}{2 P}\left[E[d]\left(1-E\left[q_{2}\right]\right)+E\left[q_{1}\right](1-E[d])\right] y^{2}\right\}+ \\
& \left.\frac{h_{1} y^{2}}{2} E\left[\delta^{2} r^{2}\right]\right\}+s I_{e}\left[\frac{1}{2} D\left(\frac{y}{P}\right)^{2}+\frac{D y}{P}\left(M-\frac{y}{P}-N\right)+\frac{1}{2} D\left(M-\frac{y}{P}-N\right)^{2}\right]+v I_{e} E[\delta](1-E[r]) y\left(M-\frac{y}{P}-N\right)-c I_{p}\left[\frac { 1 } { 2 } D \left(\frac{y}{P}+\right.\right. \\
& \left.\left.\frac{E[\delta r] y}{P_{1}}+N-M\right)^{2}+\frac{1}{2 D}\left\{\frac{(P-D-\lambda) y}{P}+\frac{\left(P_{1}-D\right) E[\delta r] y}{P_{1}}\right\}^{2}\right]
\end{aligned}
$$

By using Renewal- reward theorem, we get: 
Expected Total Profit per unit time $\left(\mathrm{E}\left[Z_{2}(y, N)\right]\right)=s D+\frac{1}{E[\alpha]}\left[-c D-i D-\frac{K D}{y}-\right.$

$\frac{h(P-D-\lambda) D y}{2 P^{2}}-\frac{h(P-D-\lambda)^{2} y}{2 P^{2}}+\frac{s I_{e} D^{2} y}{2 P^{2}}+\frac{s I_{e} D^{2}}{P}\left(M-N-\frac{y}{P}\right)+\frac{s I_{e} D^{2}}{2 y}\left(M-N-\frac{y}{P}\right)^{2}-$

$\left.\frac{c I_{p} D^{2}}{2 y}\left(\frac{y}{P}+N-M\right)^{2}-\frac{c I_{p}(P-D-\lambda)^{2} y}{2 P^{2}}\right]-\frac{E[\delta r]}{E[\alpha]}\left[(v+w) D+\frac{2 h(P-D-\lambda) D y}{P P_{1}}+\right.$

$\frac{2 h(P-D-\lambda)\left(P_{1}-D\right) D y}{P P_{1}}+\frac{c I_{p} D^{2}}{P_{1}}\left(\frac{y}{P}+N-M\right)+\frac{c I_{p}(P-D-\lambda)\left(P_{1}-D\right) y}{P P_{1}}+v I_{e} D(M-N-$

$\left.\left.\frac{y}{P}\right)\right]-\frac{E\left[\delta^{2} r^{2}\right]}{E[\alpha]}\left[\frac{h\left(P_{1}-D\right) D y}{P_{1}{ }^{2}}+\frac{h\left(P_{1}-D\right)^{2} y}{2 P_{1}{ }^{2}}+\frac{h_{1} D y}{2 P_{1}}+\frac{c I_{p} D^{2} y}{2 P_{1}{ }^{2}}+\frac{c I_{p}\left(P_{1}-D\right)^{2} y}{2 P_{1}{ }^{2}}\right]+\frac{E[\delta]}{E[\alpha]}[v D+$

$\left.v I_{e} D\left(M-N-\frac{y}{P}\right)\right]-D C_{a} \frac{E\left[d q_{2}\right]}{E[\alpha]}-D C_{r} \frac{(1-E[d]) E\left[q_{1}\right]}{E[\alpha]}-\frac{h y}{2} \frac{E\left[d q_{2} \beta\right]}{E[\alpha]}-\frac{h y}{2} \frac{E\left[d q_{2} \delta r\right]}{E[\alpha]}-$

$\frac{h D y}{2 P} \frac{\left[E[d]\left(1-E\left[q_{2}\right]\right)+E\left[q_{1}\right](1-E[d])\right]}{E[\alpha]}$

where $E[$.$] denotes the expected value.$

Case (iii) $\quad \boldsymbol{N} \leq \boldsymbol{t}_{1}+\boldsymbol{t}_{2}+\boldsymbol{N} \leq \boldsymbol{M} \leq \boldsymbol{T}+\boldsymbol{N}$

This is the case where the manufacturer earns revenue by selling the items up to $M$, beginning from time $N$. These items include the perfect items along with a proportion of reworked items and also the items disposed as scrap at $t_{1}$. He arranges for the finances to pay to the supplier for the unsold inventory lying in the period $\left(t_{1}+t_{2}+N, T+N\right)$ at some specified rate of interest as depicted in Fig. 5.

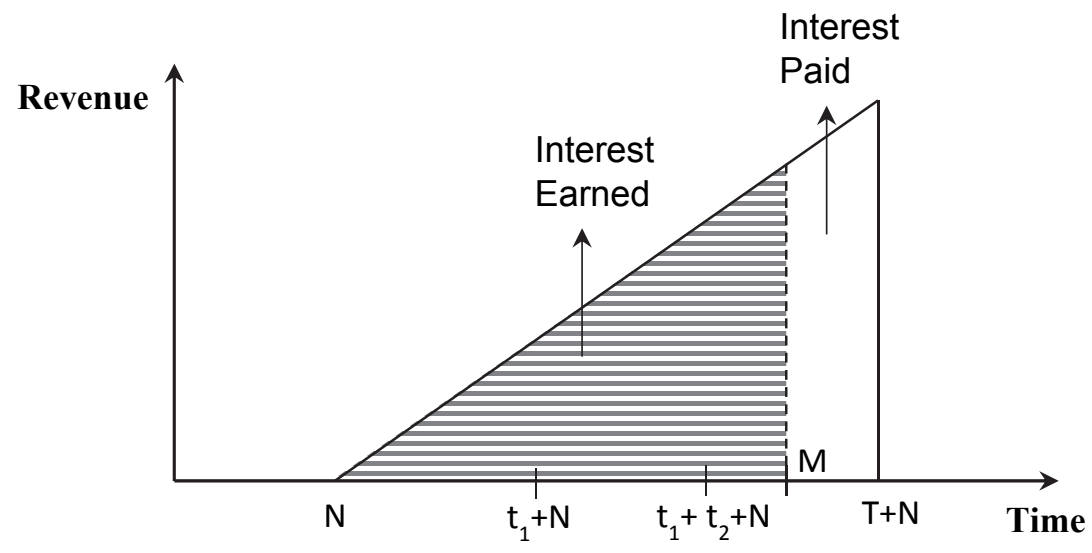

Fig. 5. $N \leq t_{1}+t_{2}+N \leq M \leq T+N$

Interest Earned $\left(\boldsymbol{I}_{e 3}\right)=s I_{e}\left[\frac{1}{2} D t_{1}{ }^{2}+D t_{1}\left(M-t_{1}-N\right)+\frac{1}{2} D t_{2}{ }^{2}+D t_{2}\left(M-t_{1}-t_{2}-\right.\right.$ $\left.N)+\frac{1}{2} D\left(M-t_{1}-t_{2}-N\right)^{2}\right]+v I_{e}\left[d+q_{1}(1-d)\right](1-r) y\left(M-t_{1}-N\right) \quad I_{e 3}=$ $S I_{e}\left[\frac{1}{2} D \frac{y^{2}}{P^{2}}+D \frac{y}{P}\left(M-\frac{y}{P}-N\right)+\frac{1}{2} D \frac{\delta^{2} r^{2} y^{2}}{P_{1}{ }^{2}}+D \frac{\delta r y}{P_{1}}\left(M-\frac{y}{P}-\frac{\delta r y}{P_{1}}-N\right)+\frac{1}{2} D\left(M-\frac{y}{P}-\right.\right.$ $\left.\left.\frac{\delta r y}{P_{1}}-N\right)^{2}\right]+v I_{e} \delta(1-r) y\left(M-\frac{y}{P}-N\right)$

Interest Payable $\left(\boldsymbol{I}_{p 3}\right)=c I_{p}\left[\frac{1}{2} D(T+N-M)^{2}\right]=c I_{p}\left[\frac{1}{2} D\left(\frac{\alpha y}{D}+N-M\right)^{2}\right]$

Total Profit (T.P.3) $=$ Total Revenue $(T . R)-$. Total Cost $($ T.C. $)+$ Interest Earned $\left(I_{e 3}\right)-$ Interest

Payable $\left(I_{p 3}\right)$ 
Total Profit $($ T.P.3 $)=\beta s y-s d q_{2} y+\delta r s y+\delta v(1-r) y-\left\{c y+K+i y+C_{r}(1-d) q_{1} y+C_{a} d q_{2} y+\right.$

$$
\begin{aligned}
& \delta w r y+h\left\{\frac{(P-D-\lambda)\left(\frac{y}{P}\right)^{2}}{2}+\left[\frac{2(P-D-\lambda) y}{P}+\left(P_{1}-D\right)\left(\frac{\delta r y}{P_{1}}\right)\right]\left(\frac{\delta r y}{P_{1}}\right)+\frac{1}{2 D}\left[\frac{(P-D-\lambda) y}{P}+\left(P_{1}-D\right)\left(\frac{\delta r y}{P_{1}}\right)\right]^{2}+\frac{d q_{2} \alpha y^{2}}{2 D}+\right. \\
& \left.\left.\frac{1}{2 P}\left[d\left(1-q_{2}\right)+q_{1}(1-d)\right] y^{2}\right\}+\frac{h_{1}}{2}(\delta r y)^{2}\right\}+ \\
& \operatorname{sI}_{e}\left[\frac{1}{2} D \frac{y^{2}}{P^{2}}+D \frac{y}{P}\left(M-\frac{y}{P}-N\right)+\frac{1}{2} D \frac{\delta^{2} r^{2} y^{2}}{P_{1}{ }^{2}}+D \frac{\delta r y}{P_{1}}\left(M-\frac{y}{P}-\frac{\delta r y}{P_{1}}-N\right)+\frac{1}{2} D\left(M-\frac{y}{P}-\frac{\delta r y}{P_{1}}-N\right)^{2}\right]+ \\
& v I_{e} \delta(1-r) y\left(M-\frac{y}{P}-N\right)-\frac{c I_{p} D}{2}\left(\frac{\alpha y}{D}+N-M\right)^{2}
\end{aligned}
$$

By using Eq. (12) and Eq. (30), we get:

$$
\begin{aligned}
& \text { Expected Total Profit }(\text { E.T.P.3 })=s y E[\beta]-s y E\left[d q_{2}\right]+s y E[\delta r]+v y E[\delta](1-E[r])-\{c y+K+ \\
& i y+C_{r} y(1-\mathrm{E}[d]) E\left[q_{1}\right]+C_{a} y E\left[d q_{2}\right]+w y E[\delta r]+h\left\{\frac{(P-D-\lambda)}{2}\left(\frac{y}{P}\right)^{2}+\left[2(P-D-\lambda) \frac{y}{P}+\left(P_{1}-\right.\right.\right. \\
& \left.\left.D)\left(\frac{y}{P_{1}}\right) E[\delta r]\right]\left(\frac{y}{P_{1}}\right) E[\delta r]\right\}+ \\
& h\left\{\frac{1}{2 D}\left[(P-D-\lambda)\left(\frac{y}{P}\right)+\left(P_{1}-D\right)\left(\frac{y}{P_{1}}\right) E[\delta r]\right]^{2}+E\left[d q_{2} \alpha\right] \frac{y^{2}}{2 D}+\frac{1}{2 P}\left[E[d]\left(1-E\left[q_{2}\right]\right)+E\left[q_{1}\right](1-E[d])\right]\right. \\
& \left.\frac{h_{1} y^{2}}{2} E\left[\delta^{2} r^{2}\right]\right\}+s I_{e}\left[\frac{1}{2} D \frac{y^{2}}{P^{2}}+D \frac{y}{P}\left(M-\frac{y}{P}-N\right)+\frac{1}{2} D \frac{E\left[\delta^{2} r^{2}\right] y^{2}}{P_{1}{ }^{2}}+D \frac{E[\delta r] y}{P_{1}}\left(M-\frac{y}{P}-\frac{E[\delta r] y}{P_{1}}-N\right)+\right. \\
& \left.\frac{1}{2} D\left(M-\frac{y}{P}-\frac{E[\delta r] y}{P_{1}}-N\right)^{2}\right]+v I_{e} E[\delta](1-E[r]) y\left(M-\frac{y}{P}-N\right)-\frac{c I_{p} D}{2}\left(\frac{E[\alpha] y}{D}+N-M\right)^{2}
\end{aligned}
$$

By using Renewal- reward theorem, we get:

Expected Total Profit per unit time $\left(\mathrm{E}\left[Z_{3}(y, N)\right]\right)=s D+\frac{1}{E[\alpha]}\left[-c D-i D-\frac{K D}{y}-\right.$

$$
\begin{aligned}
& \frac{h(P-D-\lambda) D y}{2 P^{2}}-\frac{h(P-D-\lambda)^{2} y}{2 P^{2}}+\frac{s I_{e} D^{2} y}{2 P^{2}}+\frac{S I_{e} D^{2}}{P}\left(M-N-\frac{y}{P}\right)+\frac{s I_{e} D^{2}}{2 y}\left(M-N-\frac{y}{P}\right)^{2}- \\
& \left.\frac{c I_{p} D^{2}(N-M)^{2}}{2 y}\right]-\frac{E[\delta r]}{E[\alpha]}\left[(v+w) D+\frac{2 h(P-D-\lambda) D y}{P P_{1}}+\frac{2 h(P-D-\lambda)\left(P_{1}-D\right) D y}{P P_{1}}+v I_{e} D(M-N-\right. \\
& \left.\left.\frac{y}{P}\right)\right]-\frac{E\left[\delta^{2} r^{2}\right]}{E[\alpha]}\left[\frac{h\left(P_{1}-D\right) D y}{P_{1}^{2}}+\frac{h\left(P_{1}-D\right)^{2} y}{2 P_{1}^{2}}+\frac{h_{1} D y}{2 P_{1}}\right]+\frac{E[\delta]}{E[\alpha]}\left[v D+v I_{e} D\left(M-N-\frac{y}{P}\right)\right]-D C_{a} \frac{E\left[d q_{2}\right]}{E[\alpha]}- \\
& D C_{r} \frac{(1-E[d]) E\left[q_{1}\right]}{E[\alpha]}-\frac{h y}{2} \frac{E\left[d q_{2} \beta\right]}{E[\alpha]}-\frac{h y}{2} \frac{E\left[d q_{2} \delta r\right]}{E[\alpha]}-\frac{h D y}{2 P} \frac{E\left[d\left(1-q_{2}\right)+q_{1}(1-d)\right]}{E[\alpha]}-\frac{c I_{p} y}{2} E[\alpha]-c I_{p} D(N-
\end{aligned}
$$

M)

where $E[$.$] denotes the expected value.$

\section{Case (iv) $\quad T+N \leq M$}

As explains the Fig. 6, this is the case of larger interest period resulting in no interest paid by the manufacturer to the supplier. He not only earns interest on the sales revenue generated by the selling of perfect and reworked items as per demand from time $N$ to $T$ but also an additional interest from the sale of defective lot for the time period $\left(t_{1}, M\right)$ and on the whole lot for the time period $(T, M)$. 


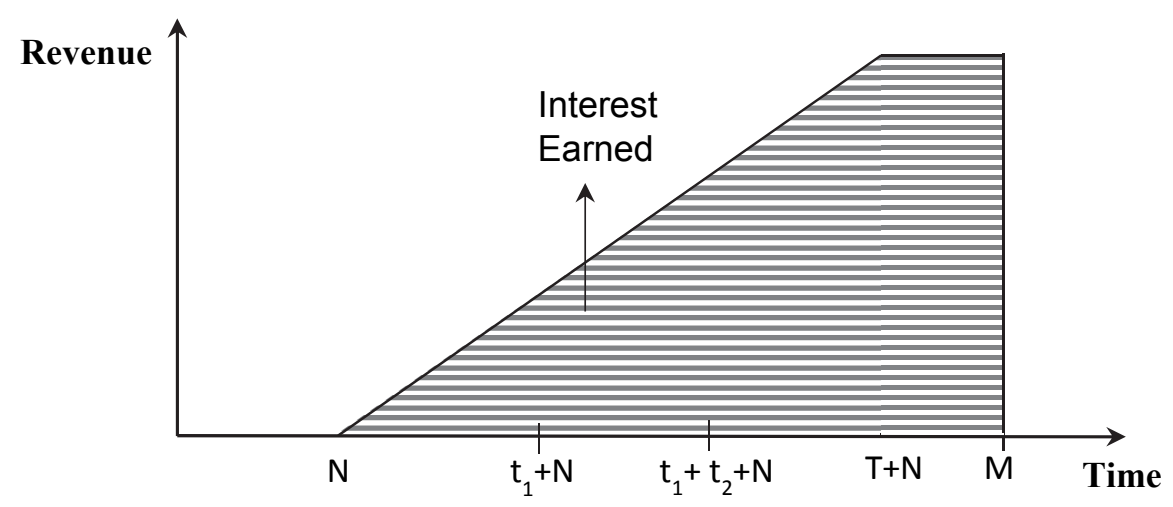

Fig. 6. $T+N \leq M$

Interest Earned $\left(I_{e} 4\right)=s I_{e}\left[\frac{1}{2} D t_{1}{ }^{2}+D t_{1}\left(M-t_{1}-N\right)+\frac{1}{2} D t_{2}{ }^{2}+D t_{2}\left(M-t_{1}-t_{2}-N\right)+\frac{1}{2} D t_{3}{ }^{2}+D t_{3}(M-\right.$ $\left.T-N)]+v I_{e}\left[d+q_{1}(1-d)\right](1-r) y\left(M-t_{1}-N\right)\right] \quad\left(I_{e q}\right)=s I_{e}\left[\frac{1}{2} D \frac{y^{2}}{P^{2}}+D \frac{y}{P}\left(M-N-\frac{y}{P}\right)+\right.$ $\frac{1}{2} D \frac{\delta^{2} r^{2} y^{2}}{P_{1}{ }^{2}}+D \frac{\delta r y}{P_{1}}\left(M-\frac{y}{P}-\frac{\delta r y}{P_{1}}-N\right)+\frac{1}{2 D}\left\{(P-D-\lambda) \frac{y}{P}+\left(P_{1}-D\right) \frac{\delta r y}{P_{1}}\right\}^{2}+\left\{(P-D-\lambda) \frac{y}{P}+\left(P_{1}-\right.\right.$ D) $\left.\left.\frac{\delta r y}{P_{1}}\right\}\left(M-\frac{\alpha y}{D}-N\right)\right]+v I_{e} \delta(1-r) y\left(M-\frac{y}{P}-N\right)$

Interest Payable $\left(I_{p 4}\right)=0$

Total Profit (T.P.4) $=$ Total Revenue $(T . R)-$. Total Cost $(T . C)+$. Interest Earned $\left(I_{e} 4\right)-$ Interest Payable $\left(I_{p 4}\right)$

Total Profit $\left(\right.$ T.P.4) $=\beta s y-s d q_{2} y+\delta r s y+\delta v(1-r) y-\left\{c y+K+i y+C_{r}(1-d) q_{1} y+C_{a} d q_{2} y+\right.$ $\delta w r y+h\left\{\frac{(P-D-\lambda)\left(\frac{y}{P}\right)^{2}}{2}+\left[\frac{2(P-D-\lambda) y}{P}+\left(P_{1}-D\right)\left(\frac{\delta r y}{P_{1}}\right)\right]\left(\frac{\delta r y}{P_{1}}\right)+\frac{1}{2 D}\left[\frac{(P-D-\lambda) y}{P}+\left(P_{1}-D\right)\left(\frac{\delta r y}{P_{1}}\right)\right]^{2}+\frac{d q_{2} \alpha y^{2}}{2 D}+\right.$ $\left.\left.\frac{1}{2 P}\left[d\left(1-q_{2}\right)+q_{1}(1-d)\right] y^{2}\right\}+\frac{h_{1}}{2}(\delta r y)^{2}\right\}+$ $S I_{e}\left[\frac{1}{2} D \frac{y^{2}}{P^{2}}+D \frac{y}{P}\left(M-\frac{y}{P}-N\right)+\frac{1}{2} D \frac{\delta^{2} r^{2} y^{2}}{P_{1}{ }^{2}}+D \frac{\delta r y}{P_{1}}\left(M-\frac{y}{P}-\frac{\delta r y}{P_{1}}-N\right)+\frac{1}{2 D}\left\{(P-D-\lambda) \frac{y}{P}+\left(P_{1}-\right.\right.\right.$ D) $\left.\left.\frac{\delta r y}{P_{1}}\right\}^{2}+\left\{(P-D-\lambda) \frac{y}{P}+\left(P_{1}-D\right) \frac{\delta r y}{P_{1}}\right\}\left(M-\frac{\alpha y}{D}-N\right)\right]+v I_{e} \delta(1-r) y\left(M-\frac{y}{P}-N\right)$

By using Eq. (12) and Eq. (35), we get:

Expected Total Profit $($ E.T.P.4 $)=s y E[\beta]-s y E\left[d q_{2}\right]+s y E[\delta r]+v y E[\delta](1-E[r])-\left\{c y+K+i y+C_{r} y(1-\right.$

$$
\begin{aligned}
& \mathrm{E}[d]) E\left[q_{1}\right]+C_{a} y E\left[d q_{2}\right]+w y E[\delta r]+h\left\{\frac{(P-D-\lambda)}{2}\left(\frac{y}{P}\right)^{2}+\left[2(P-D-\lambda) \frac{y}{P}+\left(P_{1}-D\right)\left(\frac{y}{P_{1}}\right) E[\delta r]\right]\left(\frac{y}{P_{1}}\right) E[\delta r]\right\}+ \\
& h\left\{\frac{1}{2 D}\left[(P-D-\lambda)\left(\frac{y}{P}\right)+\left(P_{1}-D\right)\left(\frac{y}{P_{1}}\right) E[\delta r]\right]^{2}+E\left[d q_{2} \alpha\right] \frac{y^{2}}{2 D}+\frac{1}{2 P}\left[E[d]\left(1-E\left[q_{2}\right]\right)+E\left[q_{1}\right](1-E[d])\right] y^{2}\right\}+ \\
& \left.\frac{h_{1} y^{2}}{2} E\left[\delta^{2} r^{2}\right]\right\}+s I_{e}\left[\frac{1}{2} D \frac{y^{2}}{P^{2}}+D \frac{y}{P}\left(M-\frac{y}{P}-N\right)+\frac{1}{2} D \frac{E\left[\delta^{2} r^{2} y^{2}\right]}{P_{1}{ }^{2}}+D \frac{E[\delta r] y}{P_{1}}\left(M-\frac{y}{P}-\frac{E[\delta r] y}{P_{1}}-N\right)+\frac{1}{2 D}\left\{(P-D-\lambda) \frac{y}{P}+\right.\right. \\
& \left.\left.\left(P_{1}-D\right) \frac{E[\delta r] y}{P_{1}}\right\}^{2}+\left\{(P-D-\lambda) \frac{y}{P}+\left(P_{1}-D\right) \frac{E[\delta r] y}{P_{1}}\right\}\left(M-\frac{E[\alpha] y}{D}-N\right)\right]+v I_{e} E[\delta](1-E[r]) y\left(M-\frac{y}{P}-N\right)
\end{aligned}
$$


By using Renewal- reward theorem, we get:

Expected Total Profit per unit time $\left(\mathrm{E}\left[Z_{4}(y, N)\right]\right)=s D+\frac{1}{E[\alpha]}\left[-c D-i D-\frac{K D}{y}-\frac{h(P-D-\lambda) D y}{2 P^{2}}-\frac{h(P-D-\lambda)^{2} y}{2 P^{2}}+\right.$

$\left.\frac{s I_{e} D^{2} y}{2 P^{2}}+\frac{s I_{e} D^{2}}{P}\left(M-N-\frac{y}{P}\right)+\frac{s I_{e}(P-D-\lambda)^{2} y}{2 P^{2}}+\frac{S I_{e}(M-N) D(P-D-\lambda)}{P}\right]-\frac{E[\delta r]}{E[\alpha]}\left[(v+w) D+\frac{2 h(P-D-\lambda) D y}{P P_{1}}+\right.$

$\frac{2 h(P-D-\lambda)\left(P_{1}-D\right) D y}{P P_{1}}-\frac{s l_{e} D^{2}}{P_{1}}\left(M-N-\frac{y}{P}\right)-\frac{s l_{e}(P-D-\lambda)\left(P_{1}-D\right) y}{P P_{1}}-\frac{(M-N) D\left(P_{1}-D\right)}{P_{1}}+v I_{e} D(M-N-$

$\left.\left.\frac{y}{P}\right)\right]-\frac{E\left[\delta^{2} r^{2}\right]}{E[\alpha]}\left[\frac{h\left(P_{1}-D\right) D y}{P_{1}{ }^{2}}+\frac{h\left(P_{1}-D\right)^{2} y}{2 P_{1}{ }^{2}}+\frac{h_{1} D y}{2 P_{1}}+\frac{s I_{e} D^{2} y}{2 P_{1}{ }^{2}}-\frac{s I_{e}\left(P_{1}-D\right)^{2} y}{2 P_{1}{ }^{2}}\right]+\frac{E[\delta]}{E[\alpha]}\left[v D+v I_{e} D\left(M-N-\frac{y}{P}\right)\right]-$

$D C_{a} \frac{E\left[d q_{2}\right]}{E[\alpha]}-D C_{r} \frac{(1-E[d]) E\left[q_{1}\right]}{E[\alpha]}-\frac{h y}{2} \frac{E\left[d q_{2} \beta\right]}{E[\alpha]}-\frac{h y}{2} \frac{E\left[d q_{2} \delta r\right]}{E[\alpha]}-\frac{h D y}{2 P} \frac{\left[E[d]\left(1-E\left[q_{2}\right]\right)+E\left[q_{1}\right](1-E[d])\right]}{E[\alpha]}-\frac{(P-D-\lambda) y}{P}-$

$\frac{\left(P_{1}-D\right) y}{P} E[\delta r]$

where $E[$.$] denotes the expected value.$

Case (v) $\quad M \leq N \leq T+N$

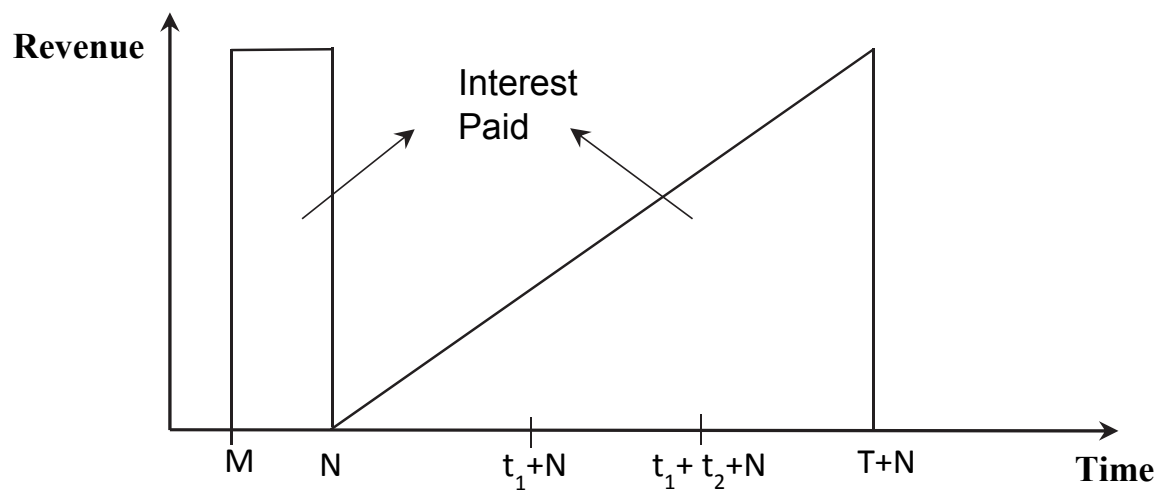

Fig. 7. $M \leq N \leq T+N$

As shown in Fig. 7, this is the case of smallest credit period where all the units are financed by the manufacturer from his own pocket, ensuring zero interest earned, to settle his account with the supplier. This is because the manufacturer gets his first payment at $N$, which happens to be after the expiration of his credit period i.e. $M$.

Interest Earned $\left(I_{e 5}\right)=0$

Interest Payable $\left(I_{p 5}\right)=c I_{p}\left[D T(N-M)+\frac{1}{2} D\left(t_{1}{ }^{2}+t_{2}{ }^{2}+t_{3}{ }^{2}\right)\right]+c I_{p}\left[d+q_{1}(1-\right.$

$d)](1-r) y\left(t_{1}+N-M\right)$

$\left(I_{p 5}\right)=c I_{p}\left\{D \frac{\alpha y}{D}(N-M)+\frac{1}{2} D \frac{y^{2}}{P^{2}}+\frac{1}{2} D \frac{\delta^{2} r^{2} y^{2}}{P_{1}{ }^{2}}+\frac{1}{2 D}\left[(P-D-\lambda) \frac{y}{P}+\left(P_{1}-D\right) \frac{\delta r y}{P_{1}}\right]^{2}\right\}+$

$c I_{p} \delta(1-r) y\left(\frac{y}{P}+M-N\right)$

Total Profit (T.P.5) $=$ Total Revenue $(T . R)-$. Total Cost $(T . C)+$. Interest Earned $\left(I_{e} 5\right)-$ Interest

Payable $\left(I_{p}\right)$ 
Total Profit $\left(\right.$ T.P.5) $=\beta s y-s d q_{2} y+\delta r s y+\delta v(1-r) y-\left\{c y+K+i y+C_{r}(1-\right.$

$$
\begin{aligned}
& \text { d) } q_{1} y+C_{a} d q_{2} y+\delta w r y+h\left\{\frac{(P-D-\lambda)\left(\frac{y}{P}\right)^{2}}{2}+\left[\frac{2(P-D-\lambda) y}{P}+\left(P_{1}-D\right)\left(\frac{\delta r y}{P_{1}}\right)\right]\left(\frac{\delta r y}{P_{1}}\right)+\right. \\
& \left.\left.\frac{1}{2 D}\left[\frac{(P-D-\lambda) y}{P}+\left(P_{1}-D\right)\left(\frac{\delta r y}{P_{1}}\right)\right]^{2}+\frac{d q_{2} \alpha y^{2}}{2 D}+\frac{1}{2 P}\left[d\left(1-q_{2}\right)+q_{1}(1-d)\right] y^{2}\right\}+\frac{h_{1}}{2}(\delta r y)^{2}\right\}- \\
& c I_{p}\left\{\alpha y(N-M)+\frac{1}{2} D \frac{y^{2}}{P^{2}}+\frac{1}{2} D \frac{\delta^{2} r^{2} y^{2}}{P_{1}^{2}}+\frac{1}{2 D}\left[(P-D-\lambda) \frac{y}{P}+\left(P_{1}-D\right) \frac{\delta r y}{P_{1}}\right]^{2}\right\}+c I_{p} \delta(1- \\
& \text { r) } y\left(\frac{y}{P}+M-N\right)
\end{aligned}
$$

By using Eq. (12) and Eq. (40), we get:

$$
\begin{aligned}
& \text { Expected Total Profit }\left(\text { E.T.P. 5) }=s y E[\beta]-s y E\left[d q_{2}\right]+s y E[\delta r]+v y E[\delta](1-E[r])-\{c y+K+i y+\right. \\
& C_{r} y(1-E[d]) E\left[q_{1}\right]+C_{a} y E\left[d q_{2}\right]+w y E[\delta r]+h\left\{\frac{(P-D-\lambda)}{2}\left(\frac{y}{P}\right)^{2}+\left[2(P-D-\lambda) \frac{y}{P}+\left(P_{1}-\right.\right.\right. \\
& \left.\left.D)\left(\frac{y}{P_{1}}\right) E[\delta r]\right]\left(\frac{y}{P_{1}}\right) E[\delta r]\right\}+ \\
& h\left\{\frac{1}{2 D}\left[(P-D-\lambda)\left(\frac{y}{P}\right)+\left(P_{1}-D\right)\left(\frac{y}{P_{1}}\right) E[\delta r]\right]^{2}+E\left[d q_{2} \alpha\right] \frac{y^{2}}{2 D}+\frac{1}{2 P}\left[E[d]\left(1-E\left[q_{2}\right]\right)+E\left[q_{1}\right](1-E[d])\right] y^{2}\right\}+ \\
& \left.\frac{h_{1} y^{2}}{2} E\left[\delta^{2} r^{2}\right]\right\}-c I_{p}\left\{\alpha y(N-M)+\frac{1}{2} D \frac{y^{2}}{P^{2}}+\frac{1}{2} D \frac{E\left[\delta^{2} r^{2}\right] y^{2}}{P_{1}{ }^{2}}+\frac{1}{2 D}\left[(P-D-\lambda) \frac{y}{P}+\left(P_{1}-D\right) \frac{E[\delta r] y}{P_{1}}\right]^{2}\right\}+c I_{p} E[\delta](1- \\
& E[r]) y\left(\frac{y}{P}+M-N\right)
\end{aligned}
$$

By using Renewal- reward theorem, we get:

Expected Total Profit per unit time $\left(\mathrm{E}\left[Z_{5}(y, N)\right]\right)=s D+\frac{1}{E[\alpha]}\left[-c D-i D-\frac{K D}{y}-\right.$

$$
\begin{aligned}
& \left.\frac{h(P-D-\lambda) D y}{2 P^{2}}-\frac{h(P-D-\lambda)^{2} y}{2 P^{2}}-\frac{c I_{p} D^{2} y}{2 P^{2}}-\frac{c I_{p} y(P-D-\lambda)^{2}}{D P^{2}}\right]-\frac{E[\delta r]}{E[\alpha]}\left[(v+w) D+\frac{2 h(P-D-\lambda) D y}{P P_{1}}+\right. \\
& \left.\frac{2 h(P-D-\lambda)\left(P_{1}-D\right) D y}{P P_{1}}+\frac{2 c I_{p} y(P-D-\lambda)\left(P_{1}-D\right)}{D P P_{1}}+c I_{p} D\left(M-N-\frac{y}{P}\right)\right]-\frac{E\left[\delta^{2} r^{2}\right]}{E[\alpha]}\left[\frac{h\left(P_{1}-D\right) D y}{P_{1}{ }^{2}}+\right. \\
& \left.\frac{h\left(P_{1}-D\right)^{2} y}{2 P_{1}{ }^{2}}+\frac{h_{1} D y}{2 P_{1}}+\frac{c I_{p} D^{2} y}{2 P_{1}{ }^{2}}+\frac{c I_{p}\left(P_{1}-D\right)^{2} y}{P_{1}{ }^{2} D}\right]+\frac{E[\delta]}{E[\alpha]}\left[v D+c I_{p} D\left(M-N-\frac{y}{P}\right)\right]-D C_{a} \frac{E\left[d q_{2}\right]}{E[\alpha]}- \\
& D C_{r} \frac{(1-E[d]) E\left[q_{1}\right]}{E[\alpha]}-\frac{h y}{2} \frac{E\left[d q_{2} \beta\right]}{E[\alpha]}-\frac{h y}{2} \frac{E\left[d q_{2} \delta r\right]}{E[\alpha]}-\frac{h D y}{2 P} \frac{\left[E[d]\left(1-E\left[q_{2}\right]\right)+E\left[q_{1}\right](1-E[d])\right]}{E[\alpha]}-C I_{p}(N-M) D
\end{aligned}
$$

Hence, the manufacturer's total profit per unit time is:

$$
Z(y, N)=\left\{\begin{array}{lr}
Z_{1}(y, N) \text { if } & N \leq M \leq t_{1}+N \leq T+N \\
Z_{2}(y, N) \text { if } & N \leq t_{1}+N \leq M \leq T+N \\
Z_{3}(y, N) \text { if } N \leq t_{1}+t_{2}+N \leq M \leq T+N \\
Z_{4}(y, N) \text { if } & T+N \leq M \\
Z_{5}(y, N) \text { if } & M \leq N \leq T+N
\end{array}\right\}
$$




\section{Optimal Solution}

In this model, the profit function, $Z(y, N)$, is a function of two decision variables out of which one is discrete, i.e., $N$, and other is continuous, i.e., $y$. In order to find the optimal values of $N$ and $y$ which jointly maximizes the expected total profit per unit time, the value of $N$ is taken as fixed.

Case wise proof of optimality is shown below.

\section{Case (i) $N \leq M \leq t_{1}+N \leq T+N$}

To determine the optimal value of $y$, say $y^{*}$, which maximizes the function of $\mathrm{E}\left[Z_{1}(y, N)\right]$ the following first-order necessary condition of optimality must be satisfied:

$\frac{\partial\left(\mathrm{E}\left[Z_{1}(y, N)\right]\right)}{\partial y}=0$ i.e.

First we partially differentiate $\mathrm{E}\left[Z_{1}(y, N)\right]$ with respect to $y$, using Eq. (22).

$$
\begin{aligned}
& \frac{\partial\left(E\left[Z_{1}(y, N)\right]\right)}{\partial y}=\frac{1}{E[\alpha]}\left[\frac{K D}{y^{2}}-\frac{h(P-D-\lambda) D}{2 P^{2}}-\frac{h(P-D-\lambda)^{2}}{2 P^{2}}-\frac{S I_{e} D^{2}(M-N)^{2}}{2 y^{2}}-\frac{c I_{p} D^{2}}{2 P^{2}}+\frac{c I_{p} D^{2}(N-M)^{2}}{2 y^{2}}-\right. \\
& \left.\frac{c I_{p}(P-D-\lambda)^{2}}{2 P^{2}}\right]-\frac{E[\delta r]}{E[\alpha]}\left[\frac{2 h(P-D-\lambda) D}{P P_{1}}+\frac{2 h(P-D-\lambda)\left(P_{1}-D\right) D}{P P_{1}}+\frac{c I_{p}(P-D-\lambda)\left(P_{1}-D\right)}{P P_{1}}-\frac{c D I_{p}}{P}\right]- \\
& \frac{E\left[\delta^{2} r^{2}\right]}{E[\alpha]}\left[\frac{h\left(P_{1}-D\right) D}{P_{1}{ }^{2}}+\frac{h\left(P_{1}-D\right)^{2}}{2 P_{1}^{2}}+\frac{h_{1} D}{2 P_{1}}+\frac{c I_{p} D^{2}}{2 P_{1}{ }^{2}}+\frac{c I_{p}\left(P_{1}-D\right)^{2}}{2 P_{1}{ }^{2}}\right]-\frac{E[\delta]}{E[r]}\left(\frac{c D I_{p}}{P}\right)-\frac{h}{2} \frac{E\left[d q_{2} \delta r\right]}{E[\alpha]}- \\
& \frac{h}{2} \frac{E\left[d q_{2} \beta\right]}{E[\alpha]}-\frac{h D}{P} \frac{E[d]\left(1-E\left[q_{2}\right]\right)+E\left[q_{1}\right](1-E[d])}{E[\alpha]}
\end{aligned}
$$

On setting Eq. (43) equal to zero, we get the optimal production size $y^{*}$ as:

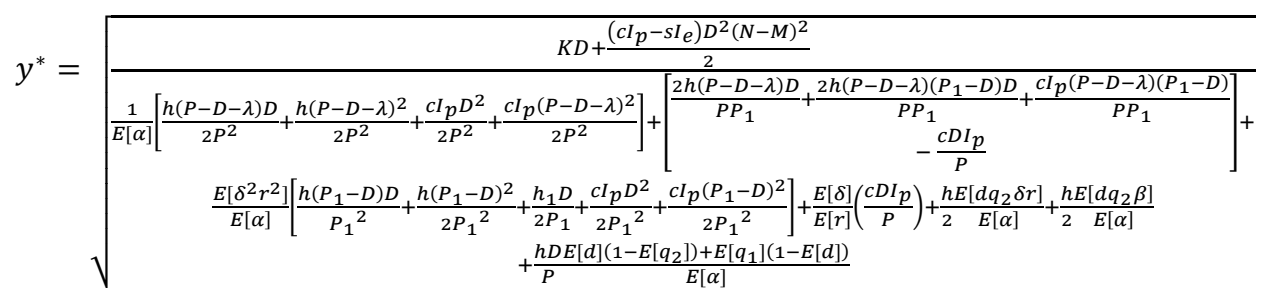

Further, it may be observed that when $d, q_{1}, q_{2, r}$ are just known values rather than random variables and when $N$ is fixed, then $y^{*}$ can be computed from: $Z_{1}^{\prime}(y, N)=0$ i.e.

$$
\begin{aligned}
& \frac{1}{\alpha}\left[\frac{K D}{y^{2}}-\frac{h(P-D-\lambda) D}{2 P^{2}}-\frac{h(P-D-\lambda)^{2}}{2 P^{2}}-\frac{s I_{e} D^{2}(M-N)^{2}}{2 y^{2}}-\frac{c I_{p} D^{2}}{2 P^{2}}+\frac{c I_{p} D^{2}(N-M)^{2}}{2 y^{2}}-\frac{c I_{p}(P-D-\lambda)^{2}}{2 P^{2}}\right]- \\
& \frac{\delta r}{\alpha}\left[\frac{2 h(P-D-\lambda) D}{P P_{1}}+\frac{2 h(P-D-\lambda)\left(P_{1}-D\right) D}{P P_{1}}+\frac{c I_{p}(P-D-\lambda)\left(P_{1}-D\right)}{P P_{1}}-\frac{c D I_{p}}{P}\right]-\frac{\delta^{2} r^{2}}{\alpha}\left[\frac{h\left(P_{1}-D\right) D}{P_{1}{ }^{2}}+\frac{h\left(P_{1}-D\right)^{2}}{2 P_{1}{ }^{2}}+\right. \\
& \left.\frac{h_{1} D}{2 P_{1}}+\frac{c I_{p} D^{2}}{2 P_{1}{ }^{2}}+\frac{c I_{p}\left(P_{1}-D\right)^{2}}{2 P_{1}{ }^{2}}\right]-\frac{\delta}{r}\left(\frac{c D I_{p}}{P}\right)-\frac{h}{2} \frac{d q_{2} \delta r}{\alpha}-\frac{h}{2} \frac{d q_{2} \beta}{\alpha}-\frac{h D}{P} \frac{d\left(1-q_{2}\right)+q_{1}(1-d)}{\alpha}=0
\end{aligned}
$$

On setting Eq. (45) equal to zero, we get the optimal production size $y^{*}$ as:

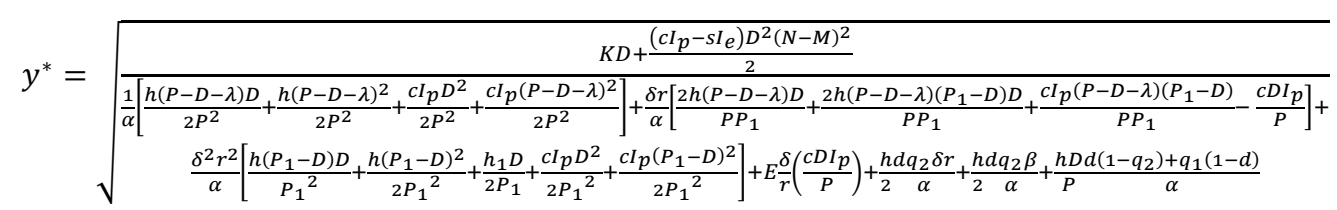


Further, to prove the concavity of the expected profit function, the following second-order sufficient condition of optimality must hold:

$\frac{\partial^{2}\left(\mathrm{E}\left[Z_{1}(y, N)\right]\right)}{\partial y^{2}} \leq 0$

By taking second order derivative of $\mathrm{E}\left[Z_{1}(y, N)\right]$ with respect to $y$, we obtain

$\frac{\partial^{2}\left(E\left[Z_{1}(y, N)\right]\right)}{\partial y^{2}}=\frac{1}{E[\alpha]}\left[-\frac{2 K D}{y^{3}}+\frac{s I_{e} D^{2}(M-N)^{2}}{y^{3}}-\frac{c I_{p} D^{2}(N-M)^{2}}{y^{3}}\right]$

For satisfying the condition of optimality,

$$
\frac{\partial^{2}\left(E\left[Z_{1}(y, N)\right]\right)}{\partial y^{2}}=\frac{1}{E[\alpha]}\left[-\frac{2 K D}{y^{3}}+\frac{\left(s I_{e}-c I_{p}\right) D^{2}(M-N)^{2}}{y^{3}}\right] \leq 0
$$

i.e. $\boldsymbol{s} \boldsymbol{I}_{\boldsymbol{e}}-\boldsymbol{c I} \boldsymbol{p} \leq \frac{\boldsymbol{2 K D}}{\boldsymbol{D}^{2}(\boldsymbol{M}-\boldsymbol{N})^{2}}$; true for fixed value of $N$ also.

\section{Case (ii) $N \leq t_{1}+N \leq M \leq T+N$}

To determine the optimal value of $y$, say $y^{*}$, which maximizes the function of $\mathrm{E}\left[Z_{2}(y, N)\right]$, the following first-order necessary condition of optimality must be satisfied:

$\frac{\partial\left(\mathrm{E}\left[Z_{2}(y, N)\right]\right)}{\partial y}=0$ i.e.

First we partially differentiate $\mathrm{E}\left[Z_{2}(y, N)\right]$ with respect to $y$, using Eq. (27).

$$
\begin{aligned}
& \frac{\partial\left(E\left[Z_{2}(y, N)\right]\right)}{\partial y}=\frac{1}{E[\alpha]}\left[\frac{K D}{y^{2}}-\frac{h(P-D-\lambda) D}{2 P^{2}}-\frac{h(P-D-\lambda)^{2}}{2 P^{2}}-\frac{s I_{e} D^{2}(M-N)^{2}}{2 y^{2}}-\frac{c I_{p} D^{2}}{2 P^{2}}+\frac{c I_{p} D^{2}(N-M)^{2}}{2 y^{2}}-\right. \\
& \left.\frac{c I_{p}(P-D-\lambda)^{2}}{2 P^{2}}\right]-\frac{E[\delta r]}{E[\alpha]}\left[\frac{2 h(P-D-\lambda) D}{P P_{1}}+\frac{2 h(P-D-\lambda)\left(P_{1}-D\right) D}{P P_{1}}-\frac{v I_{e} D}{P}+\frac{c I_{p} D^{2}}{P P_{1}}+\frac{c I_{p}(P-D-\lambda)\left(P_{1}-D\right)}{P P_{1}}\right]- \\
& \frac{E\left[\delta^{2} r^{2}\right]}{E[\alpha]}\left[\frac{h\left(P_{1}-D\right) D}{P_{1}{ }^{2}}+\frac{h\left(P_{1}-D\right)^{2}}{2 P_{1}{ }^{2}}+\frac{h_{1} D}{2 P_{1}}+\frac{c I_{p} D^{2}}{2 P_{1}{ }^{2}}+\frac{c I_{p}\left(P_{1}-D\right)^{2}}{2 P_{1}{ }^{2}}\right]-\frac{E[\delta]}{E[r]}\left(\frac{v I_{e} D}{P}\right)-\frac{h}{2} \frac{E\left[d q_{2} \delta r\right]}{E[\alpha]}-\frac{h}{2} \frac{E\left[d q_{2} \beta\right]}{E[\alpha]}- \\
& \frac{h D}{P} \frac{E[d]\left(1-E\left[q_{2}\right]\right)+E\left[q_{1}\right](1-E[d])}{E[\alpha]}
\end{aligned}
$$

On setting Eq. (48) equal to zero, we get the optimal production size $y^{*}$ as:

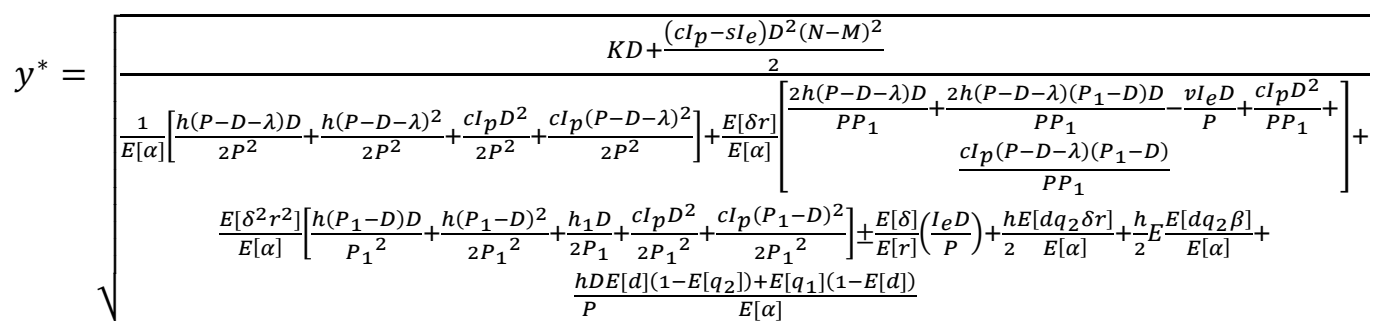

Further, it may be observed that when $d, q_{1}, q_{2}, r$ are just known values rather than random variables and when $N$ is fixed, then $y^{*}$ can be computed from: $Z_{2}^{\prime}(y, N)=0$ i. e.

$$
\begin{aligned}
& \frac{1}{\alpha}\left[\frac{K D}{y^{2}}-\frac{h(P-D-\lambda) D}{2 P^{2}}-\frac{h(P-D-\lambda)^{2}}{2 P^{2}}-\frac{s I_{e} D^{2}(M-N)^{2}}{2 y^{2}}-\frac{c I_{p} D^{2}}{2 P^{2}}+\frac{c I_{p} D^{2}(N-M)^{2}}{2 y^{2}}-\frac{c I_{p}(P-D-\lambda)^{2}}{2 P^{2}}\right]- \\
& \frac{\delta r}{\alpha}\left[\frac{2 h(P-D-\lambda) D}{P P_{1}}+\frac{2 h(P-D-\lambda)\left(P_{1}-D\right) D}{P P_{1}}-\frac{v I_{e} D}{P}+\frac{c I_{p} D^{2}}{P P_{1}}+\frac{c I_{p}(P-D-\lambda)\left(P_{1}-D\right)}{P P_{1}}\right]-\frac{\delta^{2} r^{2}}{\alpha}\left[\frac{h\left(P_{1}-D\right) D}{P_{1}{ }^{2}}+\right. \\
& \left.\frac{h\left(P_{1}-D\right)^{2}}{2 P_{1}{ }^{2}}+\frac{h_{1} D}{2 P_{1}}+\frac{c I_{p} D^{2}}{2 P_{1}{ }^{2}}+\frac{c I_{p}\left(P_{1}-D\right)^{2}}{2 P_{1}{ }^{2}}\right]-\frac{\delta}{r}\left(\frac{v I_{e} D}{P}\right)-\frac{h}{2} \frac{d q_{2} \delta r}{\alpha}-\frac{h}{2} \frac{d q_{2} \beta}{\alpha}-\frac{h D}{P} \frac{d\left(1-q_{2}\right)+q_{1}(1-d)}{\alpha}=0
\end{aligned}
$$


On setting Eq. (50) equal to zero, we get the optimal production size $y^{*}$ as:

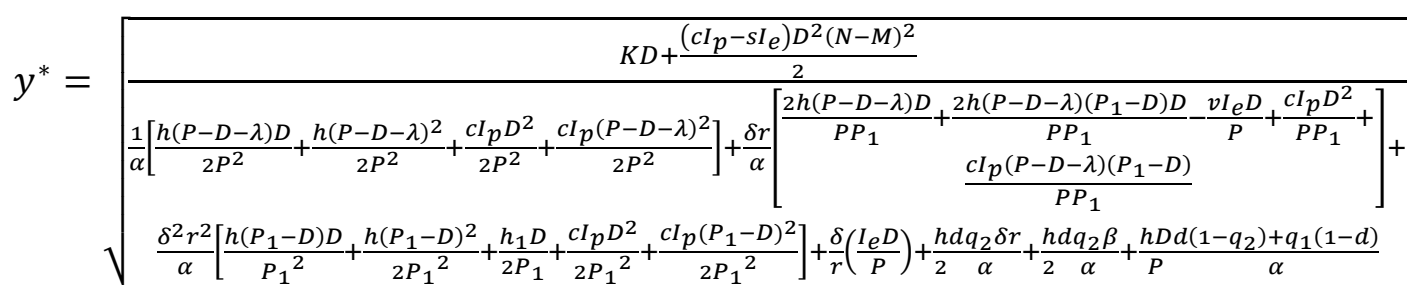

Further, to prove the concavity of the expected profit function, the following second-order sufficient condition of optimality must hold:

$\frac{\partial^{2}\left(\mathrm{E}\left[Z_{2}(y, N)\right]\right)}{\partial y^{2}} \leq 0$

By taking second order derivative of $\mathrm{E}\left[Z_{2}(y, N)\right]$ with respect to $y$, we obtain

$\frac{\partial^{2}\left(E\left[Z_{2}(y, N)\right]\right)}{\partial y^{2}}=\frac{1}{E[\alpha]}\left[-\frac{2 K D}{y^{3}}+\frac{s I_{e} D^{2}(M-N)^{2}}{y^{3}}-\frac{c I_{p} D^{2}(N-M)^{2}}{y^{3}}\right]$

For satisfying the condition of optimality,

$\frac{\partial^{2}\left(E\left[Z_{2}(y, N)\right]\right)}{\partial y^{2}}=\frac{1}{E[\alpha]}\left[-\frac{2 K D}{y^{3}}+\frac{\left(s I_{e}-c I_{p}\right) D^{2}(M-N)^{2}}{y^{3}}\right] \leq 0$

i.e.

$\boldsymbol{s} \boldsymbol{I}_{e}-\boldsymbol{C I _ { p }} \leq \frac{2 K D}{D^{2}(M-N)^{2}} ;$ true for fixed value of $N$ also

Case (iii) $N \leq t_{1}+t_{2}+N \leq M \leq T+N$

To determine the optimal value of $y$, say $y^{*}$, which maximizes the function of $\mathrm{E}\left[Z_{3}(y, N)\right]$, the following first-order necessary condition of optimality must be satisfied:

$\frac{\partial\left(\mathrm{E}\left[Z_{3}(y, N)\right]\right)}{\partial y}=0$ i.e.

First we partially differentiate $\mathrm{E}\left[Z_{3}(y, N)\right]$ with respect to $y$, using Eq. (32).

$$
\begin{aligned}
& \frac{1}{E[\alpha]}\left[\frac{K D}{y^{2}}-\frac{h(P-D-\lambda) D}{2 P^{2}}-\frac{h(P-D-\lambda)^{2}}{2 P^{2}}-\frac{S I_{e} D^{2}(M-N)^{2}}{2 y^{2}}+\frac{c I_{p} D^{2}(N-M)^{2}}{2 y^{2}}\right] \\
& -\frac{E[\delta r]}{E[\alpha]}\left[\frac{2 h(P-D-\lambda) D}{P P_{1}}+\frac{2 h(P-D-\lambda)\left(P_{1}-D\right) D}{P P_{1}}-\frac{v I_{e} D}{P}\right]-\frac{E\left[\delta^{2} r^{2}\right]}{E[\alpha]}\left[\frac{h\left(P_{1}-D\right) D}{P_{1}{ }^{2}}+\frac{h\left(P_{1}-D\right)^{2}}{2 P_{1}{ }^{2}}+\frac{h_{1} D}{2 P_{1}}\right]- \\
& \frac{E[\delta]}{E[r]}\left(\frac{v I_{e} D}{P}\right)-\frac{h}{2} \frac{E\left[d q_{2} \delta r\right]}{E[\alpha]}-\frac{h}{2} \frac{E\left[d q_{2} \beta\right]}{E[\alpha]}-\frac{h D}{P} \frac{E[d]\left(1-E\left[q_{2}\right]\right)+E\left[q_{1}\right](1-E[d])}{E[\alpha]}-\frac{c I_{p}}{2} E[\alpha]=0
\end{aligned}
$$

On setting Eq. (53) equal to zero, we get the optimal production size $y^{*}$ as:

$$
y^{*}=\sqrt{\frac{K D+\frac{\left(c p_{p}-s I_{e}\right) D^{2}(N-M)^{2}}{2}}{\frac{1}{E[\alpha]}\left[\frac{h(P-D-\lambda) D}{2 P^{2}}+\frac{h(P-D-\lambda)^{2}}{2 P^{2}}+\frac{S I_{e} D^{2}(M-N)^{2}}{2 y^{2}}-\frac{C I_{p} D^{2}(N-M)^{2}}{2 y^{2}}\right]+\frac{E[\delta r]}{E[\alpha]}\left[\frac{2 h(P-D-\lambda) D}{P P_{1}}+\frac{2 h(P-D-\lambda)\left(P_{1}-D\right) D}{P P_{1}}-\frac{v I_{e} D}{P}\right]+}}
$$

Further, it may be observed that when $d, q_{1}, q_{2}, r$ are just known values rather than random variables and when $N$ is fixed, then $y^{*}$ can be computed from: $Z_{3}^{\prime}(y, N)=0$ i.e. 


$$
\begin{aligned}
& \frac{1}{\alpha}\left[\frac{K D}{y^{2}}-\frac{h(P-D-\lambda) D}{2 P^{2}}-\frac{h(P-D-\lambda)^{2}}{2 P^{2}}-\frac{S I_{e} D^{2}(M-N)^{2}}{2 y^{2}}+\frac{c I_{p} D^{2}(N-M)^{2}}{2 y^{2}}\right] \\
& -\frac{\delta r}{\alpha}\left[\frac{2 h(P-D-\lambda) D}{P P_{1}}+\frac{2 h(P-D-\lambda)\left(P_{1}-D\right) D}{P P_{1}}-\frac{v I_{e} D}{P}\right]-\frac{\delta^{2} r^{2}}{\alpha}\left[\frac{h\left(P_{1}-D\right) D}{P_{1}{ }^{2}}+\frac{h\left(P_{1}-D\right)^{2}}{2 P_{1}^{2}}+\frac{h_{1} D}{2 P_{1}}\right]-\frac{\delta}{r}\left(\frac{v I_{e} D}{P}\right)- \\
& \frac{h}{2} \frac{d q_{2} \delta r}{\alpha}-\frac{h}{2} \frac{d q_{2} \beta}{\alpha}-\frac{h D}{P} \frac{d\left(1-q_{2}\right)+q_{1}(1-d)}{\alpha}-\frac{c I_{p}}{2} \alpha=0
\end{aligned}
$$

On setting Eq. (55) equal to zero, we get the optimal production size $y^{*}$ as:

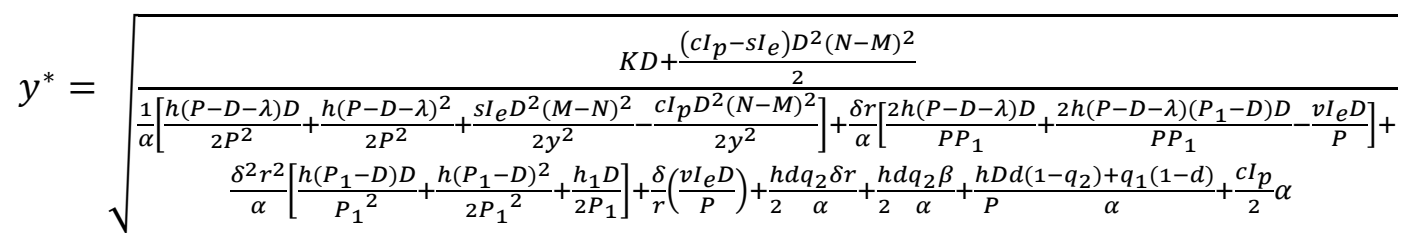

Further, to prove the concavity of the expected profit function, the following second-order sufficient condition of optimality must hold:

$\frac{\partial^{2}\left(\mathrm{E}\left[Z_{3}(y, N)\right]\right)}{\partial y^{2}} \leq 0$

By taking second order derivative of $\mathrm{E}\left[Z_{3}(y, N)\right]$ with respect to $y$, we obtain

$\frac{\partial^{2}\left(\mathrm{E}\left[Z_{3}(y, N)\right]\right)}{\partial y^{2}}=\frac{1}{E[\alpha]}\left[-\frac{2 K D}{y^{3}}+\frac{s I_{e} D^{2}(M-N)^{2}}{y^{3}}-\frac{c I_{p} D^{2}(N-M)^{2}}{y^{3}}\right]$

For satisfying the condition of optimality,

$\frac{\partial^{2}\left(\mathrm{E}\left[Z_{3}(y, N)\right]\right)}{\partial y^{2}}=\frac{1}{E[\alpha]}\left[-\frac{2 K D}{y^{3}}+\frac{\left(s I_{e}-c I_{p}\right) D^{2}(M-N)^{2}}{y^{3}}\right] \leq 0$

i.e.

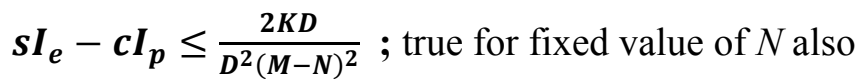

\section{Case (iv) $T+N \leq M$}

To determine the optimal value of $y$, say $y^{*}$, which maximizes the function of $\mathrm{E}\left[Z_{4}(y, N)\right]$, the following first-order necessary condition of optimality must be satisfied:

$\frac{\partial\left(E\left[Z_{4}(y, N)\right]\right)}{\partial y}=0$ i.e.

First we partially differentiate $\mathrm{E}\left[Z_{4}(y, N)\right]$ with respect to $y$, using Eq. (37).

$$
\begin{aligned}
& \frac{1}{E[\alpha]}\left[\frac{K D}{y^{2}}-\frac{h(P-D-\lambda) D}{2 P^{2}}-\frac{h(P-D-\lambda)^{2}}{2 P^{2}}+\frac{s I_{e} D^{2}}{2 P^{2}}-\frac{s I_{e} D^{2}}{P^{2}}+\frac{s I_{e}(P-D-\lambda)^{2}}{2 P^{2}}\right] \\
& -\frac{E[\delta r]}{E[\alpha]}\left[\frac{2 h(P-D-\lambda) D}{P P_{1}}+\frac{2 h(P-D-\lambda)\left(P_{1}-D\right) D}{P P_{1}}+\frac{s I_{e} D^{2}}{P_{1} P}-\frac{s I_{e}(P-D-\lambda)\left(P_{1}-D\right)}{P P_{1}}-\frac{v I_{e} D}{P}\right]- \\
& \frac{E\left[\delta^{2} r^{2}\right]}{E[\alpha]}\left[\frac{h\left(P_{1}-D\right) D}{P_{1}{ }^{2}}+\frac{h\left(P_{1}-D\right)^{2}}{2 P_{1}{ }^{2}}+\frac{h_{1} D}{2 P_{1}}+\frac{s I_{e} D^{2}}{2 P_{1}{ }^{2}}-\frac{s I_{e}\left(P_{1}-D\right)^{2}}{2 P_{1}{ }^{2}}\right]-\frac{E[\delta]}{E[\alpha]}\left(\frac{v I_{e} D}{P}\right)-\frac{h}{2} \frac{E\left[d q_{2} \delta r\right]}{E[\alpha]}-\frac{h}{2} \frac{E\left[d q_{2} \beta\right]}{E[\alpha]}- \\
& \frac{h D}{P} \frac{E[d]\left(1-E\left[q_{2}\right]\right)+E\left[q_{1}\right](1-E[d])}{E[\alpha]}-\frac{(P-D-\lambda)}{P}-\frac{\left(P_{1}-D\right)}{P} E[\delta r]=0
\end{aligned}
$$

On setting Eq. (58) equal to zero, we get the optimal production size $y^{*}$ as: 


$$
\begin{aligned}
& y^{*}=\sqrt{\frac{K D}{\frac{1}{E[\alpha]}\left[\frac{h(P-D-\lambda) D}{2 P^{2}}+\frac{h(P-D-\lambda)^{2}}{2 P^{2}}-\frac{s I_{e} D^{2}}{2 P^{2}}+\frac{s I_{e} D^{2}}{P^{2}}-\frac{s I_{e}(P-D-\lambda)^{2}}{2 P^{2}}\right]+\frac{E[\delta r]}{E[\alpha]}\left[\begin{array}{l}
\frac{2 h(P-D-\lambda) D}{P P_{1}}+\frac{2 h(P-D-\lambda)\left(P_{1}-D\right) D}{P P_{1}} \\
+\frac{s I_{e} D^{2}}{P_{1} P}-\frac{s I_{e}(P-D-\lambda)\left(P_{1}-D\right)}{P P_{1}}-\frac{v I_{e} D}{P}
\end{array}\right]+}} \\
& \frac{E\left[\delta^{2} r^{2}\right]}{E[\alpha]}\left[\frac{h\left(P_{1}-D\right) D}{P_{1}{ }^{2}}+\frac{h\left(P_{1}-D\right)^{2}}{2 P_{1}{ }^{2}}+\frac{h_{1} D}{2 P_{1}}+\frac{s I_{e} D^{2}}{2 P_{1}{ }^{2}}-\frac{s I_{e}\left(P_{1}-D\right)^{2}}{2 P_{1}{ }^{2}}\right]+\frac{E[\delta]}{E[\alpha]}\left(\frac{v I_{e} D}{P}\right)+\frac{h E\left[d q_{2} \delta r\right]}{2[\alpha]}+\frac{h E\left[d q_{2} \beta\right]}{2[\alpha]}+ \\
& \frac{h D E[d]\left(1-E\left[q_{2}\right]\right)+E\left[q_{1}\right](1-E[d])}{P}+\frac{(P-D-\lambda)}{P}+\frac{\left(P_{1}-D\right)}{P} E[\delta r]
\end{aligned}
$$

Further, it may be observed that when $d, q_{1}, q_{2}, r$ are just known values rather than random variables and when $N$ is fixed, then $y^{*}$ can be computed from: $Z_{4}^{\prime}(y, N)=0$ i.e.

$$
\begin{aligned}
& \frac{1}{\alpha}\left[\frac{K D}{y^{2}}-\frac{h(P-D-\lambda) D}{2 P^{2}}-\frac{h(P-D-\lambda)^{2}}{2 P^{2}}+\frac{s I_{e} D^{2}}{2 P^{2}}-\frac{s I_{e} D^{2}}{P^{2}}+\frac{s I_{e}(P-D-\lambda)^{2}}{2 P^{2}}\right] \\
& -\frac{\delta r}{\alpha}\left[\frac{2 h(P-D-\lambda) D}{P P_{1}}+\frac{2 h(P-D-\lambda)\left(P_{1}-D\right) D}{P P_{1}}+\frac{s I_{e} D^{2}}{P_{1} P}-\frac{s I_{e}(P-D-\lambda)\left(P_{1}-D\right)}{P P_{1}}-\frac{v I_{e} D}{P}\right]-\frac{\delta^{2} r^{2}}{\alpha}\left[\frac{h\left(P_{1}-D\right) D}{P_{1}{ }^{2}}+\right. \\
& \left.\frac{h\left(P_{1}-D\right)^{2}}{2 P_{1}{ }^{2}}+\frac{h_{1} D}{2 P_{1}}+\frac{S I_{e} D^{2}}{2 P_{1}{ }^{2}}-\frac{S I_{e}\left(P_{1}-D\right)^{2}}{2 P_{1}{ }^{2}}\right]-\frac{\delta}{\alpha}\left(\frac{v I_{e} D}{P}\right)-\frac{h}{2} \frac{d q_{2} \delta r}{\alpha}-\frac{h}{2} \frac{d q_{2} \beta}{\alpha}-\frac{h D}{P} \frac{d\left(1-q_{2}\right)+q_{1}(1-d)}{\alpha}- \\
& \frac{(P-D-\lambda)}{P}-\frac{\left(P_{1}-D\right)}{P} \delta r=0
\end{aligned}
$$

On setting Eq. (60) equal to zero, we get the optimal production size $y^{*}$ as:

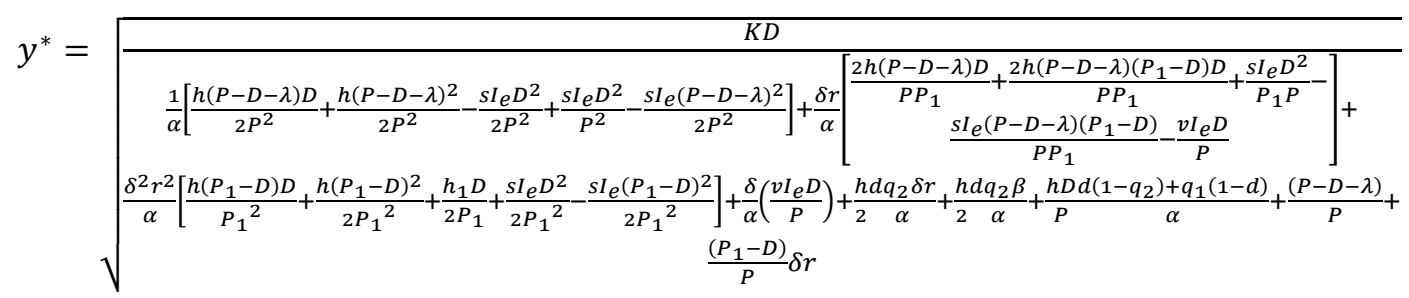

Further, to prove the concavity of the expected profit function, the following second-order sufficient condition of optimality must hold:

$\frac{\partial^{2}\left(\mathrm{E}\left[Z_{4}(y, N)\right]\right)}{\partial y^{2}} \leq 0$

By taking second order derivative of $\mathrm{E}\left[Z_{4}(y, N)\right]$ with respect to $y$, we obtain

$$
\frac{\partial^{2}\left(\mathrm{E}\left[Z_{4}(y, N)\right]\right)}{\partial y^{2}}=\frac{1}{E[\alpha]}\left[-\frac{2 K D}{y^{3}}\right]
$$

therefore $\frac{\partial^{2}\left(\mathrm{E}\left[Z_{4}(y, N)\right]\right)}{\partial y^{2}} \leq 0$; true for fixed value of $N$ also.

\section{Case (v) $M \leq N \leq T+N$}

To determine the optimal value of $y$, say $y^{*}$, which maximizes the function of $\mathrm{E}\left[Z_{5}(T, N)\right]$, the following first-order necessary condition of optimality must be satisfied:

$\frac{\partial\left(\mathrm{E}\left[Z_{5}(y, N)\right]\right)}{\partial y}=0$ i.e.

First we partially differentiate $\mathrm{E}\left[Z_{5}(y, N)\right]$ with respect to $y$, using Eq. (42). 


$$
\begin{aligned}
& \frac{1}{E[\alpha]}\left[\frac{K D}{y^{2}}-\frac{h(P-D-\lambda) D}{2 P^{2}}-\frac{h(P-D-\lambda)^{2}}{2 P^{2}}-\frac{c I_{p} D^{2}}{2 P^{2}}-\frac{c I_{p} y\left(\begin{array}{c}
P-D \\
-\lambda
\end{array}\right)^{2}}{D P^{2}}\right] \\
& -\frac{E[\delta r]}{E[\alpha]}\left[\frac{2 h(P-D-\lambda) D}{P P_{1}}+\frac{2 h(P-D-\lambda)\left(P_{1}-D\right) D}{P P_{1}}+\frac{2 c I_{p}(P-D-\lambda)\left(P_{1}-D\right)}{D P P_{1}}-\frac{c I_{p} D}{P}\right]-\frac{E\left[\delta^{2} r^{2}\right]}{E[\alpha]}\left[\frac{h\left(P_{1}-D\right) D}{P_{1}{ }^{2}}+\right. \\
& \left.\frac{h\left(P_{1}-D\right)^{2}}{2 P_{1}{ }^{2}}+\frac{h_{1} D}{2 P_{1}}+\frac{c I_{p} D^{2}}{2 P_{1}{ }^{2}}+\frac{c I_{p}\left(P_{1}-D\right)^{2}}{P_{1}{ }^{2} D}\right]-\frac{E[\delta]}{E[\alpha]}\left(\frac{c I_{p} D}{P}\right)-\frac{h}{2} \frac{E\left[d q_{2} \delta r\right]}{E[\alpha]}-\frac{h}{2} \frac{E\left[d q_{2} \beta\right]}{E[\alpha]}- \\
& \frac{h D}{P} \frac{E[d]\left(1-E\left[q_{2}\right]\right)+E\left[q_{1}\right](1-E[d])}{E[\alpha]}=0
\end{aligned}
$$

On setting Eq. (63) equal to zero, we get the optimal production size $y^{*}$ as:

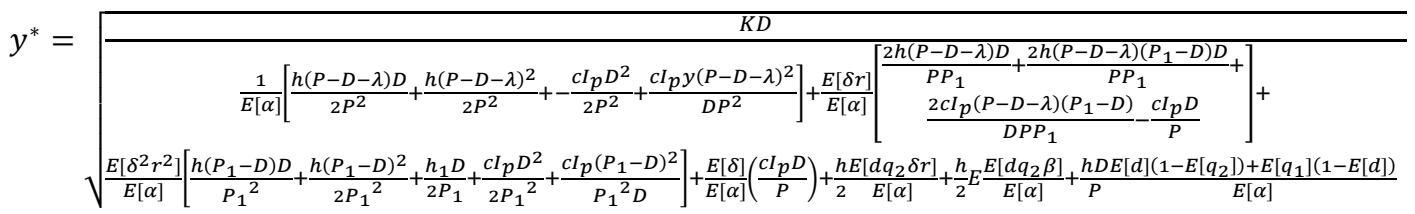

Further, it may be observed that when $d, q_{1}, q_{2}, r$ are just known values rather than random variables and when $N$ is fixed, then $y^{*}$ can be computed from: $Z_{5}^{\prime}(y, N)=0$ i.e.

$$
\begin{aligned}
& \frac{1}{\alpha}\left[\frac{K D}{y^{2}}-\frac{h(P-D-\lambda) D}{2 P^{2}}-\frac{h(P-D-\lambda)^{2}}{2 P^{2}}-\frac{c I_{p} D^{2}}{2 P^{2}}-\frac{c I_{p} y(P-D-\lambda)^{2}}{D P^{2}}\right] \\
& -\frac{\delta r}{\alpha}\left[\frac{2 h(P-D-\lambda) D}{P P_{1}}+\frac{2 h(P-D-\lambda)\left(P_{1}-D\right) D}{P P_{1}}+\frac{2 c I_{p}(P-D-\lambda)\left(P_{1}-D\right)}{D P P_{1}}-\frac{c I_{p} D}{P}\right]-\frac{\delta^{2} r^{2}}{\alpha}\left[\frac{h\left(P_{1}-D\right) D}{P_{1}{ }^{2}}+\right. \\
& \left.\frac{h\left(P_{1}-D\right)^{2}}{2 P_{1}{ }^{2}}+\frac{h_{1} D}{2 P_{1}}+\frac{c I_{p} D^{2}}{2 P_{1}{ }^{2}}+\frac{c I_{p}\left(P_{1}-D\right)^{2}}{P_{1}{ }^{2} D}\right]-\frac{\delta}{\alpha}\left(\frac{c I_{p} D}{P}\right)-\frac{h}{2} \frac{d q_{2} \delta r}{\alpha}-\frac{h}{2} \frac{d q_{2} \beta}{\alpha}-\frac{h D}{P} \frac{d\left(1-q_{2}\right)+q_{1}(1-d)}{\alpha}=0
\end{aligned}
$$

On setting Eq. (65) equal to zero, we get the optimal production size $y^{*}$ as:

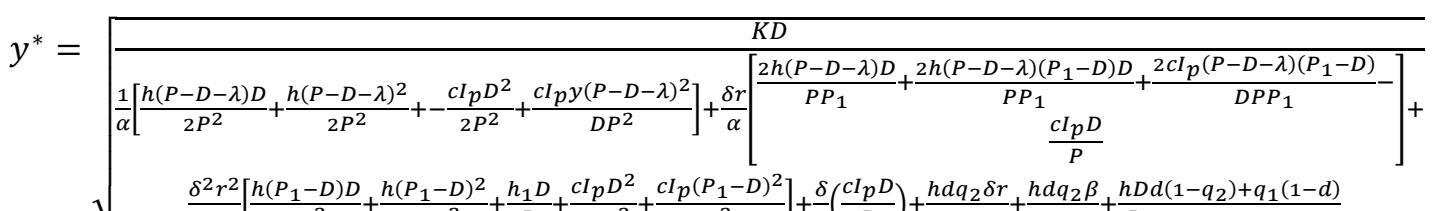

Further, to prove the concavity of the expected profit function, the following second-order sufficient condition of optimality must hold:

$\frac{\partial^{2}\left(\mathrm{E}\left[Z_{5}(y, N)\right]\right)}{\partial y^{2}} \leq 0$

By taking second order derivative of $\mathrm{E}\left[Z_{5}(T, N)\right]$ with respect to $y$, we obtain

$$
\frac{\partial^{2}\left(E\left[Z_{5}(y, N)\right]\right)}{\partial y^{2}}=\frac{1}{E[\alpha]}\left[-\frac{2 K D}{y^{3}}\right]
$$

Therefore, $\frac{\partial^{2}\left(E\left[Z_{5}(y, N)\right]\right)}{\partial y^{2}} \leq 0$, true for fixed value of $N$ also. 


\section{Special Cases}

To verify the formulation of present model, this section provides a general framework to various previously published articles.

a) In the existing model, if the formulation is confined to only imperfect quality and inspection errors but not rework and the production rate is assumed to be infinite, then the model reduces to Jaber et al (2011) model.

i.e. Setting $P \rightarrow \infty, d \neq 0, q_{1} \neq 0, q_{2} \neq 0, \lambda=$ constant, $r=0, M=0, N=0, I_{e}=0, I_{p}=0$; this implies $\delta=d+q_{1}(1-d), \beta=d q_{2}+(1-d)\left(1-q_{1}\right), \alpha=d q_{2}+(1-d)\left(1-q_{1}\right)$.

Then equations (44), (49), (54), (59), (64) can simplify to:

$$
y^{*}=\sqrt{\frac{2 K D}{h E[\mathrm{~d}] E\left[\mathrm{q}_{2}\right](1-E[\mathrm{~d}])\left(1-E\left[\mathrm{q}_{1}\right]\right)+h D\left(\frac{2}{\lambda}-\frac{D}{\lambda^{2}}+\frac{E\left[A^{2}\right]}{D}\right)}}
$$

where $A=1-\frac{D}{\lambda}-\left(d+q_{1}\right)+d\left(q_{1}+q_{2}\right)$

b) Suppose the assumption of imperfect quality is removed from the model but the concept of trade credit with credit-linked demand function is still applied. Also if the production rate approaches to infinity, then there is no production of imperfect quality items and hence the model reduces to the EOQ model of Jaggi et al (2008).

i.e. Setting $P \rightarrow \infty, d=0, q_{1}=0, q_{2}=0, r=0$; this implies $\lambda=0, \delta=0, \beta=1, \alpha=1$.

Then equations (44), (49), (54), (59), (64) can simplify to:

$$
\begin{aligned}
& T_{1}{ }^{*}=\sqrt{\frac{2 K-D(M-N)^{2}\left(s I_{e}-c I_{p}\right)}{\left(h+c I_{p}\right)}} \\
& T_{2}{ }^{*}=\sqrt{\frac{2 K}{D\left(h+c I_{p}\right)}} \\
& T_{3}{ }^{*}=\sqrt{\frac{2 K}{D\left(h+c I_{p}\right)}}
\end{aligned}
$$

c) The traditional EOQ model with imperfect quality formulated by Salameh and Jaber (2000) can be derived from this present model by neglecting the assumptions of trade credit and inspection errors along with rework.

i.e. Setting $P \rightarrow \infty, d \neq 0, q_{1}=0, q_{2}=0, r=0, M=0, N=0, I_{e}=0, I_{p}=0$; this implies $\lambda=0, \delta$

$=d, \beta=1-d, \alpha=1-d$.

$$
y^{*}=\sqrt{\frac{2 K D E\left[\frac{1}{1-d}\right]}{h\left[1-E[d]-\frac{D}{\lambda}\left(1-E\left[\frac{1}{1-d}\right]\right)\right]}}
$$

d) In the present model, if the assumption of imperfect quality is relaxed and trade credit is also removed from it, then there will be no rework done and the formulation reduces to that of classical EPQ model.

i.e. Setting $d=0, q_{1}=0, q_{2}=0, r=0, M=0, N=0, I_{e}=0, I_{p}=0$; this implies $\lambda=0, \delta=0, \beta$ $=1, \alpha=1$.

Then Eq. (45), Eq. (50), Eq. (55), Eq. (60) and Eq. (65) can simplify to:

$$
y^{*}=\sqrt{\frac{2 K D P}{h(P-D)}}
$$




\section{Algorithm}

In order to jointly optimize $y$ and $N$, the following algorithm has been proposed.

Step 0: Input all the parameters.

Step 1: Put $N=1$.

Step 2: Determine the optimal values of $T$ (i.e. $T_{1} *$ or $T_{2}^{*}$ or $T_{3}{ }^{*}$ or $T_{4} *$ or $T_{5}^{*}$ ) from the values of $y$ (i.e. $y_{1} *$ or $y_{2} *$ or $y_{3} *$ or $y_{4} *$ or $y_{5} *$ ) using Eq. (38) or Eq. (43) or Eq. (48) or Eq. (53) or Eq. (58).

Step 3: If $N \leq M \leq t_{1}+N \leq T+N$, then calculate $\mathrm{E}\left[Z_{1}(y, N)\right]$, else go to Step 5.

Step 4: If $\mathrm{E}\left[Z_{l}(y, N)\right]>\mathrm{E}\left[Z_{l}(y, N-1)\right]$, increment the value of $N$ by 1 and go to Step 2, else the current value of $N$ is optimal and the corresponding values of $T$ and $\mathrm{E}\left[Z_{1}(y, N)\right]$ can be calculated. Go to step 13 .

Step 5: If $N \leq t_{1}+N \leq M \leq T+N$, then calculate $\mathrm{E}\left[Z_{2}(y, N)\right]$, else go to Step 7 .

Step 6: If $\mathrm{E}\left[Z_{2}(y, N)\right]>\mathrm{E}\left[Z_{2}(y, N-1)\right]$, increment the value of $N$ by 1 and go to Step2, else the current value of $N$ is optimal and the corresponding values of $T$ and $\mathrm{E}\left[Z_{2}(y, N)\right]$ can be calculated. Go to step 13 .

Step 7: If $N \leq t_{1}+t_{2}+N \leq M \leq T+N$, then calculate $\mathrm{E}\left[Z_{3}(y, N)\right]$, else go to Step 9 .

Step 8: If $\mathrm{E}\left[Z_{3}(y, N)\right]>\mathrm{E}\left[Z_{3}(y, N-1)\right]$, increment the value of $N$ by 1 and go to Step2, else the current value of $N$ is optimal and the corresponding values of $T$ and $\mathrm{E}\left[Z_{3}(y, N)\right]$ can be calculated. Go to step 13 .

Step 9: If $T+N \leq M$, then calculate $\mathrm{E}\left[Z_{4}(y, N)\right]$, else go to Step 11 .

Step 10: $\mathrm{E}\left[Z_{4}(y, N)\right]>\mathrm{E}\left[Z_{4}(y, N-1)\right]$, increment the value of $N$ by 1 and go to Step2, else the current value of $N$ is optimal and the corresponding values of $T$ and $\mathrm{E}\left[Z_{4}(y, N)\right]$ can be calculated. Go to step 13.

Step 11: If $M \leq N \leq T+N$, then calculate $\mathrm{E}\left[Z_{5}(y, N)\right]$.

Step 12: $\mathrm{E}\left[Z_{5}(y, N)\right]>\mathrm{E}\left[Z_{5}(y, N-1)\right]$, increment the value of $N$ by 1 and go to Step2, else the current value of $N$ is optimal and the corresponding values of $T$ and $\mathrm{E}\left[Z_{5}(y, N)\right]$ can be calculated. Go to step 13 .

Step 13: Terminate.

\section{Numerical Example}

This section validates the model developed with the help of numerical analysis. The optimal order quantity $\left(y^{*}\right)$, retailer's credit period $\left(N^{*}\right)$, cycle length $\left(T^{*}\right)$ and expected profit per unit time $\mathrm{E}\left[Z^{*}(y, N)\right]$ are found out for a given set of parameters. Let us consider a situation with the following parameters: Now, the distributions of random variables can also be estimated from the past data. Here, it has been assumed that defect proportions $(d)$, proportion of Type-1 error $\left(q_{1}\right)$, proportion of Type-2 error $\left(q_{2}\right)$ follow Uniform distribution with their respective p.d.f. as:

$$
f(d)=\left\{\begin{array}{cc}
25, & 0 \leq d \leq 0.04 \\
0, & \text { otherwise }
\end{array}\right.
$$




$$
\begin{array}{r}
f\left(\mathrm{q}_{1}\right)=\left\{\begin{array}{lr}
50, & 0.01 \leq \mathrm{q}_{1} \leq 0.03 \\
0, & \text { otherwise }
\end{array}\right. \\
f\left(\mathrm{q}_{2}\right)=\left\{\begin{array}{lr}
25, & 0.03 \leq \mathrm{q}_{2} \leq 0.07 \\
0, & \text { otherwise }
\end{array}\right.
\end{array}
$$

With respect to rework proportion $r$ and salvage proportion (1-r), however, we assume that $r$ is fixed at 0.4 instead of being a random variable with its p.d.f. due to the computational difficulty of obtaining the result from multiple integrals involving four random variables $\left(d, q_{1}, q_{2}, r\right)$. Now, using Eqs. (75-77) and $r=0.4$, we obtain $\mathrm{E}\left[d q_{2}\right]=0.001,1 / \mathrm{E}[\alpha]=1.0233, \mathrm{E}[\delta] / \mathrm{E}[\alpha]=0.040518, \mathrm{E}\left[(\delta r)^{2}\right] / \mathrm{E}[\alpha]=0.00025674$, $E\left[d q_{2} \delta r\right] / E[\alpha]=1.6 * 10^{-5}, E\left[d q_{2} \beta\right] / E[\alpha]=9.8 * 10^{-5},\left\{\mathrm{E}[d]\left(1-\mathrm{E}\left[q_{2}\right]\right)+E\left[q_{1}\right](1-\mathrm{E}[d])\right\} /$ $E[\alpha]=0.0394$

Other parameters are: Max. demand $(U)=100$ units/day, Initial demand $(u)=30$ units/day, rate of saturation of demand $(R)=0.12, P=73,000$ units/year, $P_{l}=47,450$ units/year, $K=\$ 100 /$ production, $c$ $=\$ 25 /$ unit, $s=\$ 60 /$ unit, $v=\$ 10 /$ unit, $i=\$ 0.5 /$ unit, $C_{r}=\$ 500 /$ unit, $C_{a}=\$ 100 /$ unit, $w=\$ 5 /$ unit, $h=\$ 6 /$ unit, $h_{1}=\$ 8 /$ unit, $d=0.02, q_{1}=0.02, q_{2}=0.05, I_{e}=0.08 /$ unit/year, $I_{p}=0.14 /$ unit $/$ year, $M=10$ days $(=0.027$ years $)$

From Eq. (60), the optimal production lot size $y^{*}=\mathbf{2 , 4 0 0}$ units, $N^{*}=\mathbf{8 . 7}$ days $(=0.023$ years $), D^{*}(N)=$ $\mathbf{2 8 , 1 4 9}$ units/year, $T^{*}=\mathbf{1 0}$ days $\left(=0.027\right.$ years) with corresponding $\mathrm{E}\left[Z^{*}(\boldsymbol{y}, \boldsymbol{N})\right]=\mathbf{\$ \mathbf { 3 } , \mathbf { 7 6 } , 2 5 2} /$ year.

Also, the optimal solution validates the condition of no shortages

$$
\begin{aligned}
& \text { a. } \beta y>D t_{1} \text { Since L. H.S. }=2,307 \text { and R. H. S. }=1,158 \\
& \text { b. }\left(\beta y-D t_{1}+\delta r y\right) \geq D\left(t_{2}+t_{3}\right) \text { Since L. H.S. }=1,187 \text { and R. H.S. }=1,187
\end{aligned}
$$

\section{Significance of the strategic modeling}

In this section, we investigate the impact of two stage trade credit strategy with credit linked demand function on the existing inventory models for imperfect quality items with inspection errors. The advantage with the assimilation of two stage trade credit strategy is two folds in contrast to one stage trade credit. It not only gives positive vibes to the end customers by giving them interest free credit period, but also to the retailer by stimulating his demand. The increased demand owes to the fact that more customers are attracted to this strategy, and this justifies the purpose of taking credit-linked demand function into the modeling. The utility of this promotional tool is also supported by the numerical results.

Table 2

Impact of $M$ on optimal production policy (in the presence of 2 stage trade credit)

\begin{tabular}{lccccc}
\hline $\boldsymbol{M}$ & $\boldsymbol{N}^{*}$ (in days) & $\boldsymbol{D}^{*}(\boldsymbol{N})$ & $\boldsymbol{T}^{*}($ in days $)$ & $\boldsymbol{y}^{*}$ & $\mathbf{E}\left[\boldsymbol{Z}^{*}(\boldsymbol{y}, \boldsymbol{N})\right]$ \\
\hline $\mathbf{0}$ & 0 & 10,950 & 4 & 933 & 53,159 \\
$\mathbf{5}$ & 3 & 19,515 & 5 & 1,664 & $1,62,666$ \\
$\mathbf{1 0}$ & 8.7 & 28,149 & 10 & 2,400 & $3,76,252$ \\
$\mathbf{1 5}$ & 13.9 & 32,180 & 15 & 2,744 & $5,05,726$ \\
$\mathbf{2 0}$ & 18.9 & 34,239 & 20 & 2,919 & $5,79,165$ \\
$\mathbf{2 5}$ & 19.8 & 34,470 & 21 & 2,939 & $6,10,287$ \\
\hline
\end{tabular}

The results from Table 2 indicate that as the supplier offers the manufacturer a larger credit period, the manufacturer also offers his customer a higher credit period in order to stimulate the demand. This phenomenon results in an increase of production lot size and hence the profit for the retailer. The reason behind this practical phenomenon is the presence of two stage trade credit strategy along with creditlinked demand function, which enables the retailer to increase his profits by earning more revenue due to elevated sales, and also by gaining interest on the deposited revenue. In other words, as the length of credit period given to the customers increases, more demand is captured by the retailer, which eventually leads to higher sales revenue. Moreover, when there is no permissible delay in payment from either side i.e., $(N=0, M=0)$ as observed in Table 2 , the optimal order quantity and total profit values have gone 
steep down due to constant demand. From the above discussion, it is evident that the incorporation of trade credit has a substantial impact on the overall profit of the manufacturer. So WLOG, trade credit acts as a good business strategy which should be explored by any intelligent decision maker, so as to expand his customer base and henceforth his profit.

\section{Sensitivity Analysis}

The change in the values of parameters may happen due to uncertainties in any decision-making situations. For simplicity of analysis, we use deterministic values of $d, q_{1}, q_{2}$ and $r$ and not the expected ones. So, we determine $Z^{*}(y, N)$ instead of $\mathrm{E}\left[Z^{*}(y, N)\right], N^{*}$ and $y^{*}$ from Eq. (45), Eq. (50), Eq. (55), Eq. (60), (65) rather Eq. (44), Eq. (49), Eq. (54), Eq. (59), Eq. (64) in each case. To analyze these changes, sensitivity analysis has been performed to study the effect of changes in main parameters $M, d, q_{1}, q_{2}, r$, $R$ on the optimal production control variables $\left(y^{*} \& T^{*}\right)$ and $\mathrm{E}\left[Z^{*}(y, N)\right]$ in presence of two stage trade credit.

From the computational results in Tables 3-7, we obtain the following managerial insights:

Table 3

Impact of $d$ on optimal production policy

\begin{tabular}{llll}
\hline $\boldsymbol{d}$ & $\boldsymbol{D}^{*}(\boldsymbol{N})$ & $\boldsymbol{y}^{*}$ & $\mathbf{E}\left[\boldsymbol{Z}^{*}(\boldsymbol{y}, \boldsymbol{N})\right]$ \\
\hline $\mathbf{0 . 0 0}$ & 28,125 & 2,372 & $3,89,109$ \\
$\mathbf{0 . 0 2}$ & 28,149 & 2,400 & $3,76,252$ \\
$\mathbf{0 . 0 4}$ & 28,172 & 2,429 & $3,61,799$ \\
$\mathbf{0 . 0 6}$ & 28,196 & 2,458 & $3,46,051$ \\
$\mathbf{0 . 0 8}$ & 28,219 & 2,488 & $3,29,068$ \\
$\mathbf{0 . 1 0}$ & 28,241 & 2,519 & $3,10,732$ \\
\hline
\end{tabular}

Table 4

Impact of $q_{1}$ on optimal production policy

\begin{tabular}{llll}
\hline $\boldsymbol{q}_{\boldsymbol{1}}$ & $\boldsymbol{D} *(\boldsymbol{N})$ & $\boldsymbol{y}^{*}$ & $\mathbf{E}[\boldsymbol{Z} *(\boldsymbol{y}, \boldsymbol{N})]$ \\
\hline $\mathbf{0 . 0 1}$ & 28,136 & 2,384 & $4,00,994$ \\
$\mathbf{0 . 0 2}$ & 28,149 & 2,400 & $3,76,252$ \\
$\mathbf{0 . 0 3}$ & 28,161 & 2,416 & $3,50,634$ \\
$\mathbf{0 . 0 4}$ & 28,174 & 2,431 & $3.24,115$ \\
$\mathbf{0 . 0 5}$ & 28,186 & 2,447 & $2,96,667$ \\
$\mathbf{0 . 0 6}$ & 28,199 & 2,463 & $2,68,261$ \\
\hline
\end{tabular}

- Table 3 reflects that when the percentage of defective items $(d)$ increases, the production quantity increases while the demand and profit decreases. Such is observed due to the fact that increment in proportion of bad quality items compels the manufacturer to produce more items to meet the demand since a substantial amount is discarded as scrap which also reduces the profit margins by large extent. Also in order to withstand the competitiveness of the market, the retailer needs to look into the source of received lot, and corrective measures should be taken to improve the quality of supply as it will also help in increasing demand.

- Table 4 illustrates that increasing the proportion of Type 1 error $\left(q_{1}\right)$, increases the total number of defects by mistake, thereby refraining the manufacturer to achieve the maximum possible sales. By virtue of the fact that higher proportion is discarded as scrap and lesser quantity is sold as perfect items with the increment in proportion of $\left(q_{1}\right)$, an increasing tendency of production quantity is reflected in the table to meet the declining trend of demand. Since only some items are recovered through rework process and sold as perfect items while the remaining proportion goes to scrap, it causes reduction in value of profit in the end.

Table 5

Impact of $q_{2}$ on optimal production policy

\begin{tabular}{llll}
\hline $\boldsymbol{q}_{2}$ & $\boldsymbol{D}^{*}(\boldsymbol{N})$ & $\boldsymbol{y}^{*}$ & $\mathbf{E}\left[\boldsymbol{Z}^{*}(\boldsymbol{y}, \boldsymbol{N})\right]$ \\
\hline $\mathbf{0 . 0 1}$ & 28,152 & 2,402 & $3,86,163$ \\
$\mathbf{0 . 0 2}$ & 28,150 & 2,401 & $3,83,682$ \\
$\mathbf{0 . 0 3}$ & 28,149 & 2,401 & $3,81,203$ \\
$\mathbf{0 . 0 4}$ & 28,149 & 2,400 & $3,78,727$ \\
$\mathbf{0 . 0 5}$ & 28,149 & 2,400 & $3,76,252$ \\
$\mathbf{0 . 0 6}$ & 28,148 & 2,399 & $3,73,780$ \\
\hline
\end{tabular}

Table 6

\begin{tabular}{llll}
\multicolumn{4}{l}{ Impact of $r$ on optimal production policy } \\
\hline $\boldsymbol{r}$ & $\boldsymbol{D}^{*}(\boldsymbol{N})$ & $\boldsymbol{y}^{*}$ & $\mathbf{E}\left[\boldsymbol{Z}^{*}(\boldsymbol{y}, \boldsymbol{N})\right]$ \\
\hline $\mathbf{0 . 1}$ & 28,153 & 2,430 & $3,73,057$ \\
$\mathbf{0 . 2}$ & 28,152 & 2,420 & $3,74,136$ \\
$\mathbf{0 . 3}$ & 28,150 & 2,410 & $3,75,201$ \\
$\mathbf{0 . 4}$ & 28,149 & 2,400 & $3,76,252$ \\
$\mathbf{0 . 5}$ & 28,147 & 2,390 & $3,77,289$ \\
$\mathbf{0 . 6}$ & 28,146 & 2,380 & $3,78,314$ \\
\hline
\end{tabular}

- As evident from Table 5, an increase in Type 2 error $\left(q_{2}\right)$ leads to lowering of profit majorly due to loss of demand. Selling of defectives not only brings frustration to customers but also penalizes the manufacturer through return and goodwill losses. Though with the increase in $\left(q_{2}\right)$, a higher 
fraction of the defect returns go through the rework process but all are not sold at selling price $(s)$ due to irretrievable changes in the items and hence larger quantity is sold as scrap reducing the profit values henceforth. Decrease in the production quantity is largely because of demand depreciation

- In Table 6 it is seen that higher the percentage of items being reworked $(r)$, more is the count of items sold at selling price $(s)$ after the end of rework process. Hence, adding to the revenue directly since reworked items are treated as good as perfect items. So, it is profitable to the manufacturer to send maximum items to the rework process as it positively affects the demand also. Resultantly, the manufacturer does not need to increase the production volume since reworked items can satisfy the customer demand more in place of perfect items.

Table 7

Impact of $R$ on optimal production policy.

\begin{tabular}{llll}
\hline $\boldsymbol{R}$ & $\boldsymbol{D} *(\boldsymbol{N})$ & $\boldsymbol{y}$ & $\mathbf{E}\left[\boldsymbol{Z}^{*}(\boldsymbol{y}, \boldsymbol{N})\right]$ \\
\hline $\mathbf{0 . 0 8}$ & 23,952 & 2,042 & $2,61,588$ \\
$\mathbf{0 . 1 0}$ & 26,236 & 2,237 & $3,21,454$ \\
$\mathbf{0 . 1 2}$ & 28,149 & 2,400 & $2,76,252$ \\
$\mathbf{0 . 1 4}$ & 29,739 & 2,535 & $4,25,055$ \\
$\mathbf{0 . 1 6}$ & 31,053 & 2,648 & $4,67,622$ \\
$\mathbf{0 . 1 8}$ & 32,134 & 2,740 & $5,04,135$ \\
\hline
\end{tabular}

- It is clear from Table 7 that as the rate of saturation of demand $(R)$ increases, the demand increases indicating a rise in production quantity and profit values. Owing to the fact that increment in the value of $(R)$ is directly proportional to the retailer's credit period mathematically, so it eventually helps in increasing the demand. To satisfy the demand in a more fulfilling manner, the manufacturer needs to increase his production volume. It also allows the retailer to achieve maximum sales and hence profit is increasing with the increase of $(R)$.

Discussion on the incorporated factors

For better understanding of these varying parameters, graphs representing the relative change in the optimal values of decision variables with respect to the independent parameters are provided.

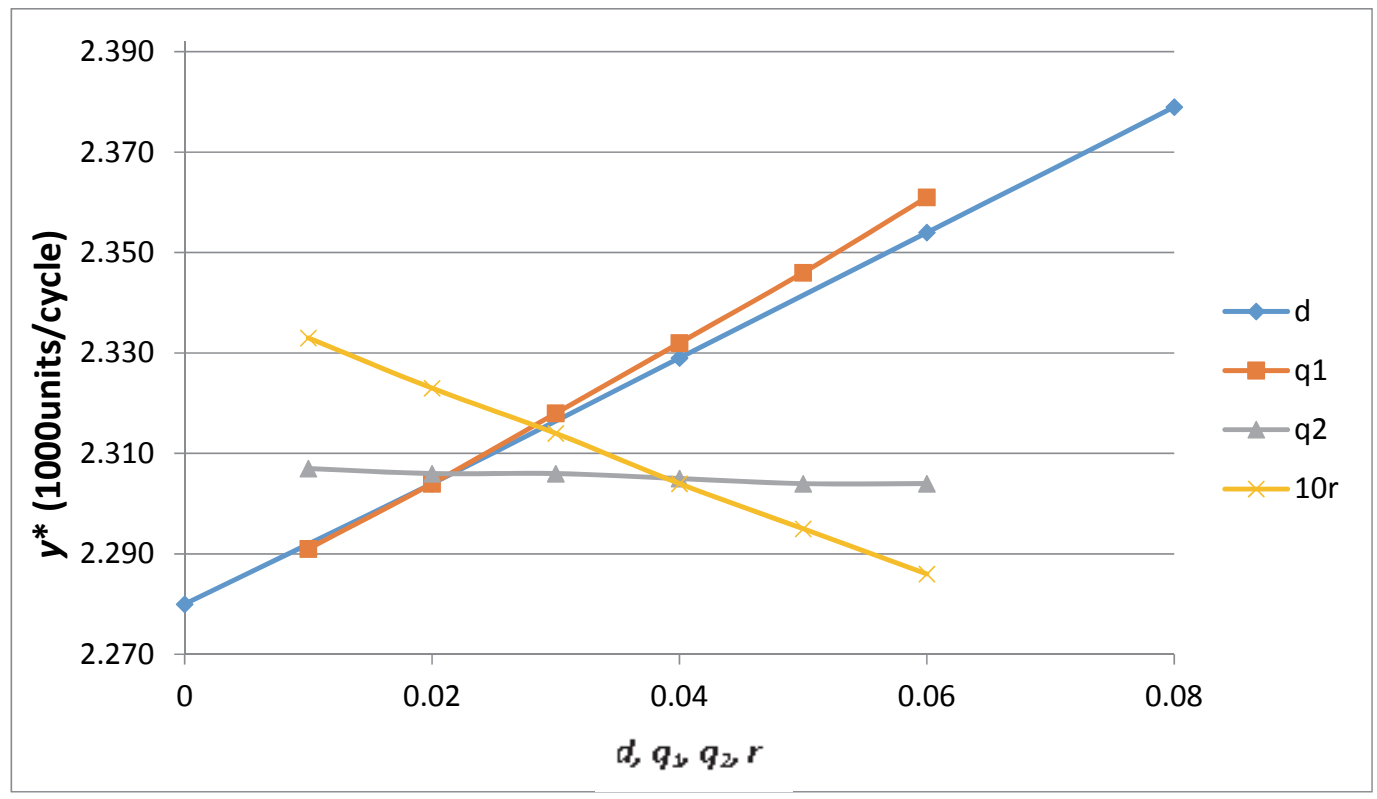

Fig. 8. Effect of increase in different parameters viz. $d, q_{1}, q_{2}, r$ on $y^{*}$ 


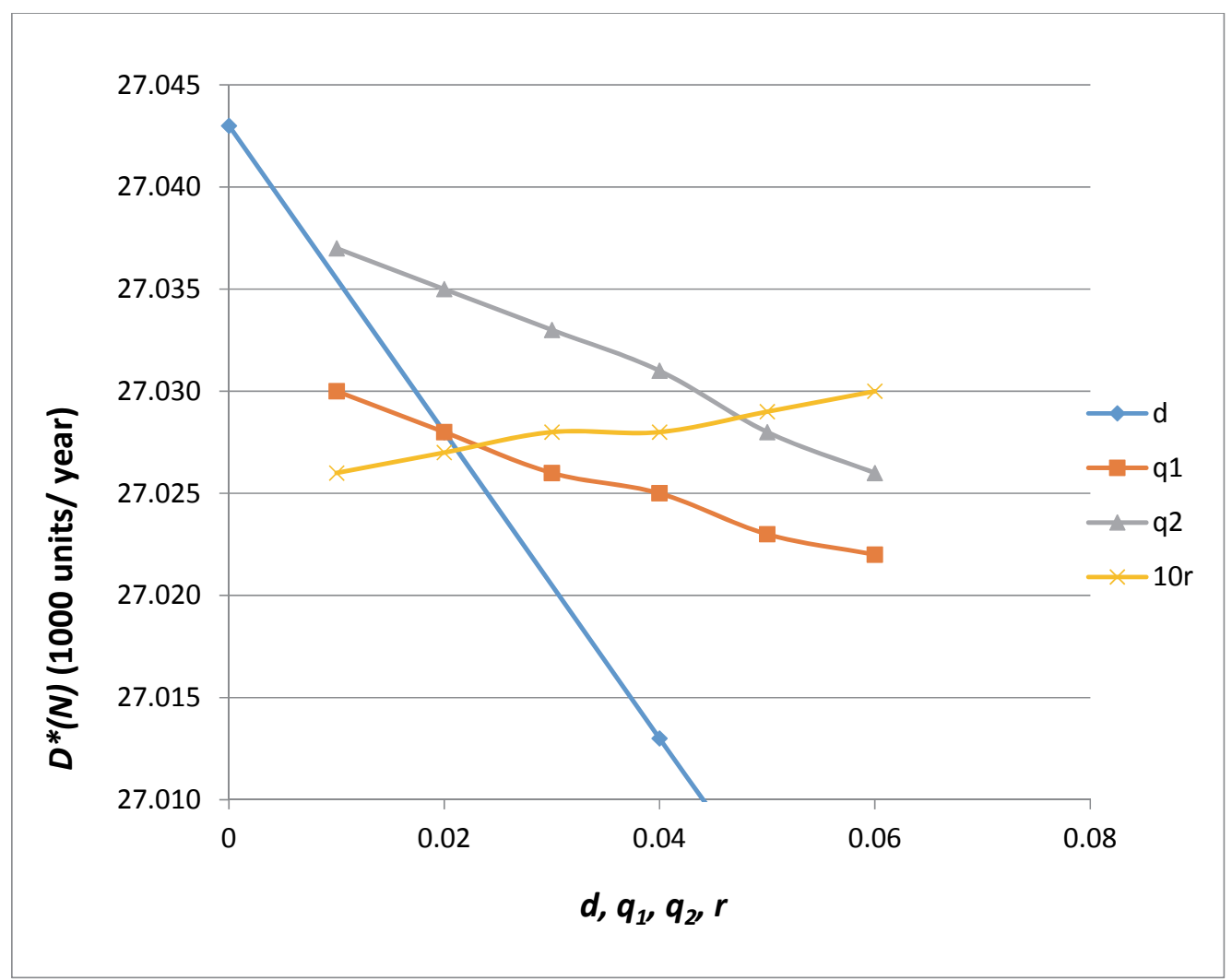

Fig. 9. Effect of increase in different parameters viz. $d, q_{1}, q_{2}, r$ on $D^{*}(N)$

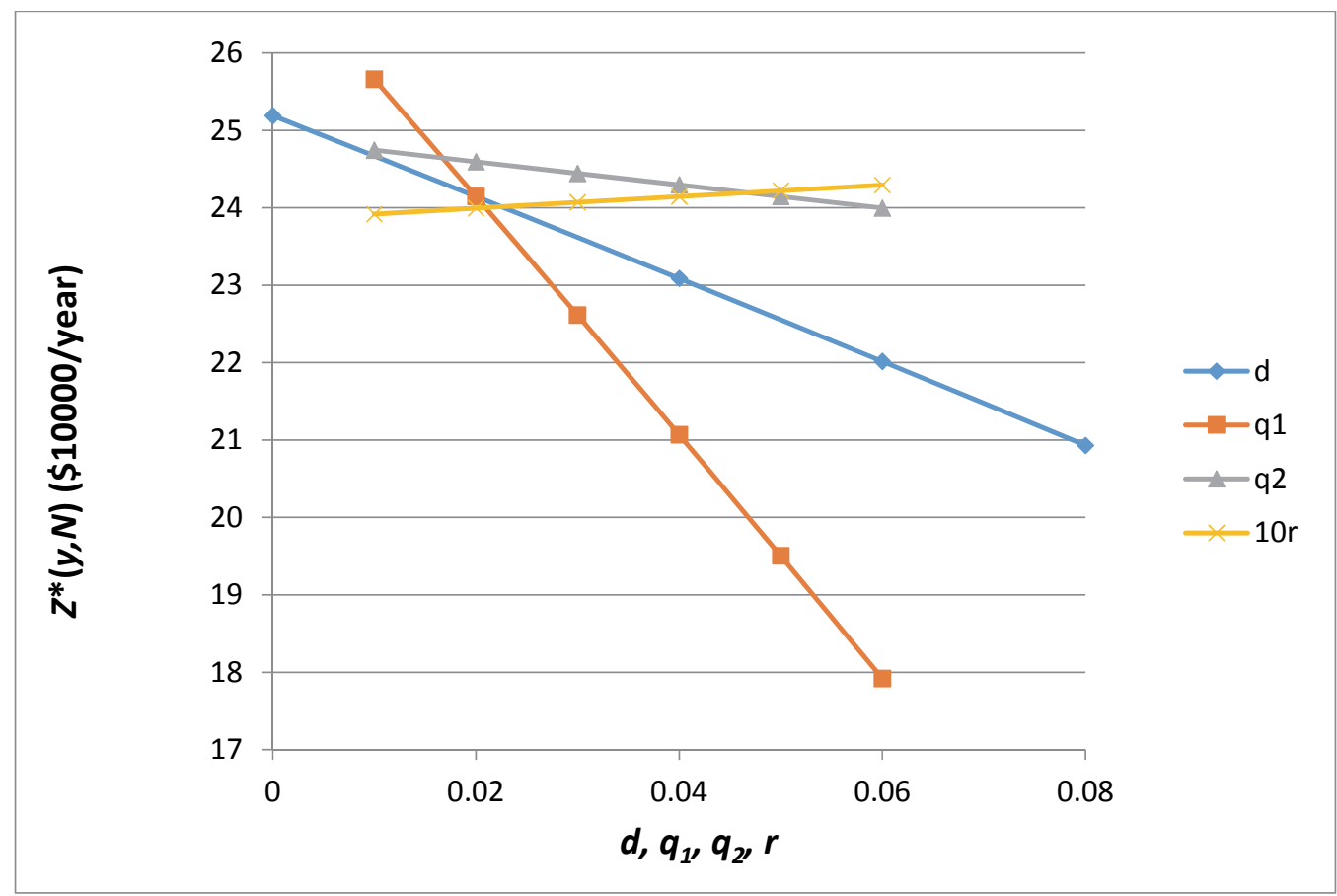

Fig. 10. Effect of increase in different parameters viz. $d, q_{1}, q 2, r$ on $\mathrm{E}\left[Z^{*}(y, N)\right]$ 


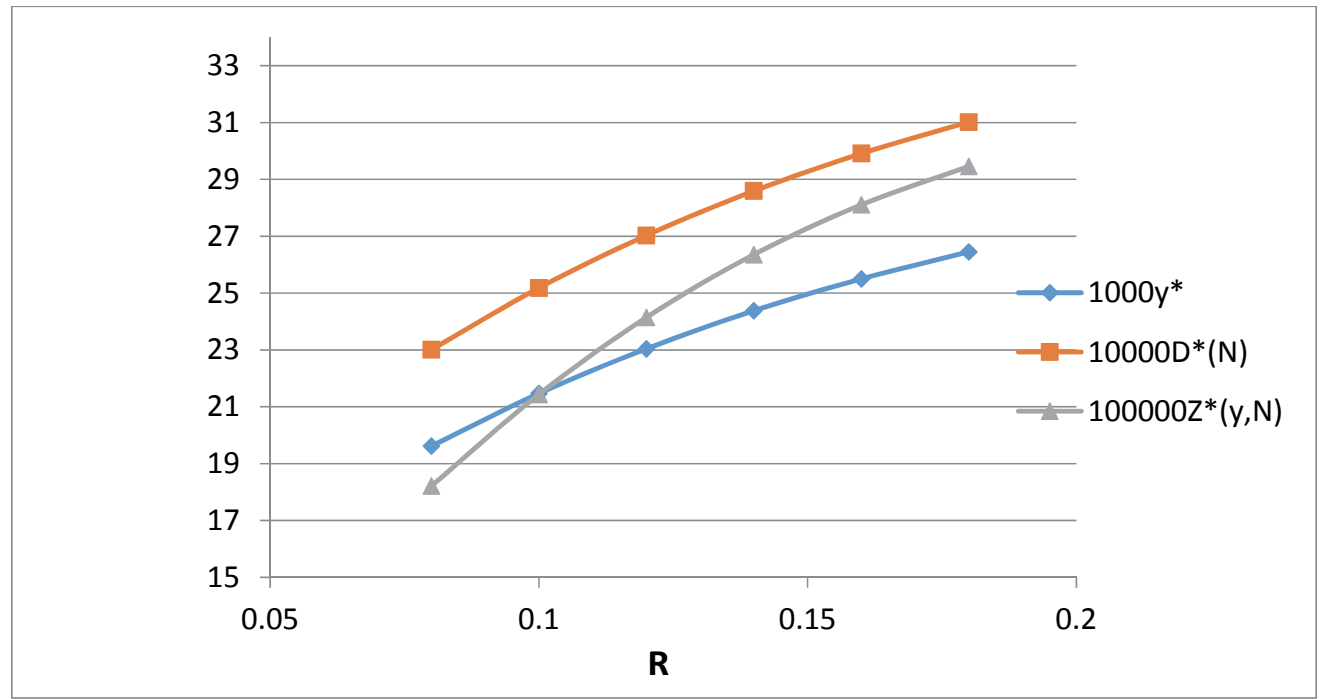

Fig. 11. Behaviour of optimal values of on $y^{*}, \mathrm{E}\left[Z^{*}(y, N)\right], D^{*}(N)$ with increase in $R$

Fig. 8 depicts the comparative behavior of different parameters considered in the model, viz. $d, q_{1}, q_{2}$ and $r$ on decision variable $y^{*}$. The contribution of $d$ is seen to be the highest in increasing the value of $y^{*}$ as compared to the remaining parameters i.e. $q_{1}, q_{2}$ and $r$. It is explained by the fact that with the increase in the supply of bad quality items, the manufacturer needs to produce more number of perfect items to satisfy the demand. Same is the reason for increase in the value of $y^{*}$ due to $q_{1}$ as it also elevates the count of scrap items only. Reverse is the effect of increase in $r$ on $y^{*}$ since reworked items add to the count of perfect items. Although no significant change is observed on $y^{*}$ due to $q_{2}$.

Fig. 9 shows the effect of increase in the values of different parameters $d, q_{1}, q_{2}$ and $r$, on decision variable $D^{*}(N)$ which is opposite to the change brought by these parameters to $y^{*}$. With the rising number of imperfect quality items due to increase in proportions of $d$ and $q_{1}$, the demand is observed to be decreasing which proves that the manufacturer cannot compromise on the quality part. Also, due to the increase in the proportions of sale of false products i.e. $q_{2}$, the demand is seen to have a downfall due to loss of goodwill which is again very difficult to recover. However, reverse trend is detected on increase in the proportion of reworked items i.e. $r$ since these help the manufacturer in raising the count of perfect quality items and hence uplifting the demand indirectly.

Fig. 10 reveals the outcome of increase in the values of different parameters $d, q_{1}, q_{2}$ and $r$, on decision variable $\mathrm{E}\left[Z^{*}(y, N)\right]$. Since total profit is directly proportional to the sale of perfect quality items hence it descends with the increase of defect related parameters i.e. $d$ and $q_{1}$. However, there is not much fall in the profit values due to increase in the proportion of sales returns i.e. $q_{2}$ since it is assumed that all the defect returns are replaced with the good quality items. Although, some decline is still exhibited in the total profit values since only a fraction of defect returns are recovered through rework process while remaining are discarded as scrap. Moreover, contrary behaviour is reflected on optimal values of $\mathrm{E}\left[Z^{*}(y, N)\right]$ with the increase in the proportion of reworked items i.e. $r$ since reworked items directly add to the sale of perfect items.

Fig. 11 exposes the effect of single parameter $R$ on decision variables $y^{*}, D^{*}(N)$ and $\mathrm{E}\left[Z^{*}(y, N)\right]$. When the rate of saturation of demand increases, it affects all the decision variables positively. This justifies the pupose of taking such a demand function which inreases with increase of $R$. Consequently, it increases the production volume to satisfy the elevated demand and hence directly raising the profit values. 


\section{Conclusion}

In the present paper, a strategic production modeling for defective items with imperfect inspection process, rework, and sales return under two-level trade credit has been developed. The model establishes the importance of trade credit strategies in the current business environment. In order to generate more revenue, both supplier and manufacturer offer a credit period to their respective customers to stimulate the demand. Some previous research articles are deduced as special cases in the present study, which also authenticates the proposed model. From the managerial perspective the present study undoubtedly represents a pragmatic business model by incorporating the concept of trade credit, which makes it more relevant and applicable in practice. The proposed model provides the following managerial implications:

- With an increase in manufacturer's credit period, customers' credit period also increases which results in higher demand, which eventually leads to a higher profit for the manufacturer.

- As the percentage of defective items increases, the production quantity increases while the demand and profit decreases.

- As the proportion of Type 1 error increases, lesser quantity is sold as perfect items, which leads to a decline in profits.

- An increase in Type 2 error leads to lowering of profit majorly due to loss of demand, since it penalizes the manufacturer through return and goodwill losses.

- The higher the percentage of items being reworked, more is the count of items sold at selling price, which adds to the revenue directly. So, it is profitable to the manufacturer to send maximum items to the rework process as it positively affects the demand also.

For future investigation, the proposed model can be extended under different realistic situations viz., multi-items in a supply chain, production model with shortages, imperfect rework process, different type of demand functions like time-varying demand, stock-dependent demand etc.

\section{Acknowledgement}

The first and third author would like to acknowledge the support of the Research Grant No.: RC/2015/9677, provided by the University of Delhi, Delhi, India for conducting this research.

\section{References}

Aggarwal, S. P., \& Jaggi, C. K. (1995). Ordering policies of deteriorating items under permissible delay in payments. Journal of the Operational Research Society, 46(5), 658-662.

Chang, C. T., Cheng, M. C., \& Soong, P. Y. (2016). Impacts of inspection errors and trade credits on the economic order quantity model for items with imperfect quality. International Journal of Systems Science: Operations \& Logistics, 3(1), 34-48.

Chiu, Y. P. (2003). Determining the optimal lot size for the finite production model with random defective rate, the rework process, and backlogging. Engineering Optimization, 35(4), 427-437.

Chiu, S. W., Ting, C. K., \& Chiu, Y. S. P. (2007). Optimal production lot sizing with rework, scrap rate, and service level constraint. Mathematical and Computer Modelling, 46(3), 535-549.

Cárdenas-Barrón, L. E. (2009). Economic production quantity with rework process at a single-stage manufacturing system with planned backorders. Computers \& Industrial Engineering, 57(3), 11051113.

Chiu, S. W. (2010). Robust planning in optimization for production system subject to random machine breakdown and failure in rework. Computers \& Operations Research, 37(5), 899-908.

Chung, K. J. (2011). The economic production quantity with rework process in supply chain management. Computers \& Mathematics with Applications, 62(6), 2547-2550.

Chung, K. J., \& Liao, J. J. (2011). The simplified solution algorithm for an integrated supplier-buyer inventory model with two-part trade credit in a supply chain system. European Journal of Operational Research, 213(1), 156-165. 
Duffuaa, S. O., \& Khan, M. (2005). Impact of inspection errors on the performance measures of a general repeat inspection plan. International Journal of Production Research, 43(23), 4945-4967.

Goyal, S. K. (1985). Economic order quantity under conditions of permissible delay in payments. Journal of the Operational Research Society, 36(4), 335-338.

Haley, C. W. \& Higgins, R. C. (1973). Inventory policy and trade financing. Management Science, 20(4), 464-471.

Hayek, P. A., \& Salameh, M. K. (2001). Production lot sizing with the reworking of imperfect quality items produced. Production Planning \& Control, 12(6), 584-590.

Hsu, J. T., \& Hsu, L. F. (2013a). An EOQ model with imperfect quality items, inspection errors, shortage backordering, and sales returns. International Journal of Production Economics, 143(1), 162-170.

Hsu, J. T., \& Hsu, L. F. (2013b). Two EPQ models with imperfect production processes, inspection errors, planned backorders, and sales returns. Computers \& Industrial Engineering, 64(1), 389-402.

Ho, C. H., Ouyang, L. Y., \& Su, C. H. (2008). Optimal pricing, shipment and payment policy for an integrated supplier-buyer inventory model with two-part trade credit. European Journal of Operational Research, 187(2), 496-510.

Jaggi, C. K., Goyal, S. K., \& Goel, S. K. (2008). Retailer's optimal replenishment decisions with creditlinked demand under permissible delay in payments. European Journal of Operational Research, 190(1), 130-135.

Jaggi, C. K., Khanna, A., \& Kishore, A. (2016). Production inventory policies for defective items with inspection errors, sales return, imperfect rework process and backorders. In Advancement in Science and Technology: Proceedings of the 2nd International Conference on Communication Systems (ICCS2015) (Vol. 1715, p. 020062). AIP Publishing.

Jaggi, C. K., Tiwari, S., \& Shafi, A. (2015). Effect of deterioration on two-warehouse inventory model with imperfect quality. Computers \& Industrial Engineering, 88, 378-385.

Jamal, A. M. M., Sarker, B. R., \& Wang, S. (1997). An ordering policy for deteriorating items with allowable shortage and permissible delay in payment. Journal of the Operational Research Society, 48(8), 826-833.

Khan, M., Jaber, M. Y., \& Bonney, M. (2011). An economic order quantity (EOQ) for items with imperfect quality and inspection errors. International Journal of Production Economics, 133(1), 113118.

Kim, J., Hwang, H., \& Shinn, S. (1995). An optimal credit policy to increase supplier's profits with pricedependent demand functions. Production Planning \& Control, 6(1), 45-50.

Kreng, V. B., \& Tan, S. J. (2011). Optimal replenishment decision in an EPQ model with defective items under supply chain trade credit policy. Expert Systems with Applications, 38(8), 9888-9899.

Lee, H. L., \& Rosenblatt, M. J. (1987). Simultaneous determination of production cycle and inspection schedules in a production system. Management Science, 33(9), 1125-1136.

Liu, N., Kim, Y., \& Hwang, H. (2009). An optimal operating policy for the production system with rework. Computers \& Industrial Engineering, 56(3), 874-887.

Ouyang, L. Y., \& Chang, C. T. (2013). Optimal production lot with imperfect production process under permissible delay in payments and complete backlogging. International Journal of Production Economics, 144(2), 610-617.

Papachristos, S., \& Konstantaras, I. (2006). Economic ordering quantity models for items with imperfect quality. International Journal of Production Economics, 100(1), 148-154.

Porteus, E. L. (1986). Optimal lot sizing, process quality improvement and setup cost reduction. Operations research, 34(1), 137-144.

Rosenblatt, M. J., \& Lee, H. L. (1986). Economic production cycles with imperfect production processes. IIE transactions, $18(1), 48-55$.

Schwaller, R.L. (1988). EOQ under inspection costs. Production and Inventory Management, 29(3), 2224.

Raouf, A., Jain, J. K., \& Sathe, P. T. (1983). A cost-minimization model for multicharacteristic component inspection. IIE Transactions, 15(3), 187-194. 
Salameh, M. K., \& Jaber, M. Y. (2000). Economic production quantity model for items with imperfect quality. International Journal of Production Economics, 64(1), 59-64.

Sana, S. S. (2010). An economic production lot size model in an imperfect production system. European Journal of Operational Research, 201(1), 158-170.

Schrady DA (1967). A deterministic inventory model for repairable items. Naval Research Logistics, 14(3), 391-398.

Sarkar, B., Cárdenas-Barrón, L. E., Sarkar, M., \& Singgih, M. L. (2014). An economic production quantity model with random defective rate, rework process and backorders for a single stage production system. Journal of Manufacturing Systems, 33(3), 423-435.

Taft, E. W. (1918). The most economical production lot. Iron Age, 101(18), 1410-1412.

Teng, J. T. (2002). On the economic order quantity under conditions of permissible delay in payments. Journal of the Operational Research Society, 53(8), 915-918.

Teng, J. T., \& Chang, C. T. (2009). Optimal manufacturer's replenishment policies in the EPQ model under two levels of trade credit policy. European Journal of Operational Research, 195(2), 358-363.

Thangam, A., \& Uthayakumar, R. (2009). Two-echelon trade credit financing for perishable items in a supply chain when demand depends on both selling price and credit period. Computers \& Industrial Engineering, 57(3), 773-786.

Tiwari, S., Cárdenas-Barrón, L. E., Khanna, A., \& Jaggi, C. K. (2016). Impact of trade credit and inflation on retailer's ordering policies for non-instantaneous deteriorating items in a two-warehouse environment.International Journal of Production Economics, 176, 154-169.

Vörös, J. (2013). Economic order and production quantity models without constraint on the percentage of defective items. Central European Journal of Operations Research, 21(4), 867-885.

Wee, H. M., \& Widyadana, G. A. (2013). A production model for deteriorating items with stochastic preventive maintenance time and rework process with FIFO rule. Omega, 41(6), 941-954.

Yoo, S. H., Kim, D., \& Park, M. S. (2009). Economic production quantity model with imperfect-quality items, two-way imperfect inspection and sales return. International Journal of Production Economics, 121(1), 255-265.

Yoo, S. H., Kim, D., \& Park, M. S. (2012). Inventory models for imperfect production and inspection processes with various inspection options under one-time and continuous improvement investment. Computers \& Operations Research, 39(9), 2001-2015.

Zhang, X., \& Gerchak, Y. (1990). Joint lot sizing and inspection policy in an EOQ model with random yield. IIE transactions, 22(1), 41-47.

Zhou, Y., Chen, C., Li, C., \& Zhong, Y. (2016). A synergic economic order quantity model with trade credit, shortages, imperfect quality and inspection errors. Applied Mathematical Modelling, 40(2), 1012-1028.

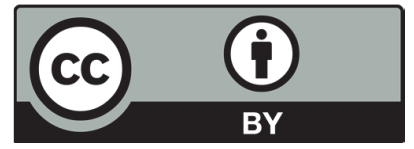

(C) 2016 by the authors; licensee Growing Science, Canada. This is an open access article distributed under the terms and conditions of the Creative Commons Attribution (CCBY) license (http://creativecommons.org/licenses/by/4.0/). 